\title{
POLYNOMIAL PARTITIONING ON VARIETIES OF CODIMENSION TWO AND POINT-HYPERSURFACE INCIDENCES IN FOUR DIMENSIONS
}

\author{
SAUGATA BASU AND MARTÍN SOMBRA
}

\begin{abstract}
We present a polynomial partitioning theorem for finite sets of points in the real locus of an irreducible complex algebraic variety of codimension at most two. This result generalizes the polynomial partitioning theorem on the Euclidean space of Guth and Katz, and its extension to hypersurfaces by Zahl and by Kaplan, Matoušek, Sharir and Safernová.

We also present a bound for the number of incidences between points and hypersurfaces in the four-dimensional Euclidean space. It is an application of our partitioning theorem together with the refined bounds for the number of connected components of a semi-algebraic set by Barone and Basu.
\end{abstract}

\section{Contents}

1. Introduction

2. Preliminaries on Hilbert functions and semi-algebraic geometry 4

3. Partitioning finite sets on varieties 10

4. Point-hypersurface incidences 13

$\begin{array}{ll}\text { References } & 22\end{array}$

\section{INTRODUCTION}

The polynomial partitioning method was introduced by Guth and Katz in their seminal paper [GK10]. Applying it in conjunction with the Elekes' framework [ES11], they made a breakthrough in a long-standing problem of Erdős on the number of distinct distances between points in the plane, by nearly proving the distinct distances conjecture. Subsequently, this method has been applied to produce other new results and simpler proofs of known results in discrete geometry, see for instance [KMS12, ST12].

The Guth-Katz polynomial partitioning method gives a nonlinear decomposition of the Euclidean space, which plays a role analogous to cuttings or trapezoidal decompositions in the more classical Clarkson-Shor type divide-and-conquer arguments for such problems, see for instance $\left[\mathrm{CEG}^{+} 90\right]$.

Date: September 22, 2015.

2010 Mathematics Subject Classification. Primary 52C10; Secondary 13D40, 14P25.

Key words and phrases. Polynomial partitioning, Hilbert functions, connected components of semi-algebraic sets, point-hypersurface incidences.

Basu and Sombra were partially supported by the IPAM research program "Algebraic Techniques for Combinatorial and Computational Geometry". Basu was also partially supported by NSF grants CCF-0915954, CCF-1319080 and DMS-1161629. Sombra was also partially supported by the MINECO research project MTM2012-38122-C03-02. 
It can be summarized in the result below. For a polynomial $g \in \mathbb{R}\left[x_{1}, \ldots, x_{d}\right]$, we denote by $V(g)$ its zero zet in $\mathbb{C}^{d}$ and, for a finite set $\mathcal{Q}$, we denote by $\operatorname{card}(\mathcal{Q})$ its cardinality.

Theorem 1.1 (Guth and Katz [GK10]). Let $d \geq 1$ and $\mathcal{P} \subset \mathbb{R}^{d}$ be a finite subset. Given $\ell \geq 1$, there is a nonzero polynomial $g \in \mathbb{R}\left[x_{1}, \ldots, x_{d}\right]$ of degree bounded by $\ell$ such that, for each connected component $C$ of $\mathbb{R}^{d} \backslash V(g)$,

$$
\operatorname{card}(\mathcal{P} \cap C)=O_{d}\left(\frac{\operatorname{card}(\mathcal{P})}{\ell^{d}}\right)
$$

where the implicit constant in the $O$-notation depends only on $d$.

When applying this result in a concrete situation, one needs to couple it with a suitable bound for the number of connected components of the semi-algebraic set $\mathbb{R}^{d} \backslash V(g)$. This is provided by the classical works of Oleŭnik, Petrovskiı̌, Milnor and Thom on the Betti numbers of semi-algebraic varieties [PO49, Mil64, Tho65], which allow to treat the points in $\mathcal{P}$ outside the hypersurface $V(g)$.

However, it is possible that many, or even all, of the points in $\mathcal{P}$ are contained in this hypersurface. The points in $\mathcal{P} \cap V(g)$ are not partitioned, and a separate argument is needed for handling them. The natural approach would be to apply a polynomial partitioning theorem on $V(g)$ together with a suitable bound for the number of connected components of the resulting partition. After this step, it is also possible that many of the points in $\mathcal{P} \cap V(g)$ are contained in the partitioning variety of codimension 2 . Then one would like to apply a partitioning theorem on this variety, and so on.

To make this strategy work efficiently, one needs a polynomial partitioning theorem on varieties. For hypersurfaces, such a result has been achieved independently by Zahl [Zah13] and by Kaplan, Matoušek, Sharir and Safernová [KMSS12], and applied to incidence problems in $\mathbb{R}^{3}$. Extending it to varieties of arbitrary codimension has been identified as a major obstacle to apply the polynomial partitioning method to incidence problems in dimension $d \geq 4$, see for instance the discussion in [KMSS12, §3]. Our main objective in this paper is to present such a result for irreducible varieties of codimension two.

Given an irreducible algebraic variety $X \subset \mathbb{C}^{d}$ we denote by $\operatorname{dim}(X)$ and $\operatorname{deg}(X)$ its dimension and degree, respectively. We also denote by $\delta(X)$ the minimal integer $\delta \geq 1$ such that $X$ is an irreducible component of the zero set of a family of polynomials of degree bounded by $\delta$. These invariants are related by the inequalities (Lemma 2.2)

$$
\delta(X) \leq \operatorname{deg}(X) \leq \delta(X)^{d-\operatorname{dim}(X)} .
$$

The following is a simplified version of our polynomial partitioning theorem (Theorem 3.1).

Theorem 1.2. Let $d \geq 1$ and $X \subset \mathbb{C}^{d}$ an irreducible variety of codimension at most two. Let $\mathcal{P} \subset \mathbb{R}^{d} \cap X$ be a finite subset and $\ell \geq 6 d \delta(X)$. Then there is a polynomial $g \in \mathbb{R}\left[x_{1}, \ldots, x_{d}\right]$ of degree bounded by $\ell$ with $\operatorname{dim}(X \cap V(g))=\operatorname{dim}(X)-1$ such that, for each connected component $C$ of $\mathbb{R}^{d} \backslash V(g)$,

$$
\operatorname{card}(\mathcal{P} \cap C)=O_{d}\left(\frac{\operatorname{card}(\mathcal{P})}{\operatorname{deg}(X) \ell^{\operatorname{dim}(X)}}\right) .
$$

When $X=\mathbb{C}^{d}$, the invariant $\delta(X)$ is equal to 1 whereas, when $X$ is a hypersurface, it coincides with $\operatorname{deg}(X)$. Hence, Theorem 1.2 reduces in these cases to 
Theorem 1.1 and to the polynomial partitioning theorems in [Zah13, KMSS12], respectively.

As for the Guth-Katz theorem, the proof of this result is based on the ham sandwich theorem obtained by Stone and Tukey from the Borsuk-Ulam theorem. The new key ingredient is the systematic use of the upper and lower bounds for Hilbert functions due to Chardin [Cha89] and Chardin and Philippon [CP99].

Remark 1.3. The polynomial partition method also applies to problems in computational geometry, in particular to range searching with semi-algebraic sets. Concurrently with this paper, Matoušek and Patáková have also obtained a polynomial partitioning theorem on varieties [MS14, Theorem 1.1], focused on obtaining efficient range searching algorithms.

For irreducible varieties of codimension two, the Matoušek-Patáková partitioning theorem is quantitatively weaker than ours. On the other hand, this result holds in a more general setting, since it can be applied to non-necessarily irreducible varieties of arbitrary dimension. This greater generality is important for their application to range searching.

As a test case for Theorem 1.2, we consider the problem of bounding the number of point-hypersurface incidences. Given a set $\mathcal{P}$ of points of $\mathbb{R}^{d}$ and a set $\mathcal{V}$ of subvarieties of $\mathbb{R}^{d}$ or of $\mathbb{C}^{d}$, we denote by $I(\mathcal{P}, \mathcal{V})$ their number of incidences, that is, the number of pairs $(p, V) \in \mathcal{P} \times \mathcal{V}$ with $p \in V$.

The following fundamental result was proved by Szemerédi and Trotter in 1983, in response to a problem of Erdős.

Theorem 1.4 (Szemerédi and Trotter [ST83]). Let $\mathcal{P}$ be a set of $m$ points of $\mathbb{R}^{2}$ and $\mathcal{L}$ a set of $n$ lines in $\mathbb{R}^{2}$. Then

$$
I(\mathcal{P}, \mathcal{L})=O\left(m^{\frac{2}{3}} n^{\frac{2}{3}}+m+n\right) .
$$

This theorem has led to an extensive study of incidences of points and curves in the plane, and of points and varieties in higher dimensions. In particular, it was extended by Pach and Sharir to incidences between points in the plane and curves having a bounded degree of freedom [PS98]. Later on, Zahl obtained an analogous result for the incidences between points in $\mathbb{R}^{3}$ and algebraic surfaces having a bounded degree of freedom [Zah13]. A similar result was independently obtained by Kaplan, Matoušek, Sharir and Safernová for the incidences between points in $\mathbb{R}^{3}$ and unit spheres [KMSS12].

We present the following bound for the number of incidences between points in $\mathbb{R}^{4}$ and threefolds.

Theorem 1.5. Given $k, c \geq 1$, let $\mathcal{P}$ be a finite set of points of $\mathbb{R}^{4}$ and $\mathcal{H}$ a finite set of hypersurfaces of $\mathbb{C}^{4}$ satisfying the following conditions:

(a) the degrees of the hypersurfaces in $\mathcal{H}$ are bounded by $c$;

(b) the intersection of any four distinct hypersurfaces in $\mathcal{H}$ is finite;

(c) for any subset of $k$ distinct points in $\mathcal{P}$, the number of hypersurfaces in $\mathcal{H}$ containing them is bounded by $c$.

Set $m=\operatorname{card}(\mathcal{P})$ and $n=\operatorname{card}(\mathcal{H})$. Then

$$
I(\mathcal{P}, \mathcal{H})=O_{k, c}\left(m^{1-\frac{k-1}{4 k-1}} n^{1-\frac{3}{4 k-1}}+m+n\right) .
$$

This result is an application of Theorem 1.2 together with the refined bounds for the number of connected components of a semi-algebraic set due to Barone and 
Basu [BB12, BB13]. Our whole approach is strongly inspired by the treatment of the unit distance problem in three dimensions in [Zah13, KMSS12].

Theorem 1.5 is a particular case of a conjectural bound for the number of pointhypersurface incidences in $\mathbb{R}^{d}$ (Conjecture 4.1). Related with this, we propose two further conjectures: a generalization of our polynomial partitioning theorem to varieties of arbitrary codimension (Conjecture 3.4) and a bound for the number of connected components of a semi-algebraic set depending on the degree of that variety, instead of the Bézout number of a set of defining equations (Conjecture 2.10). If one can show that these two conjectures are true, it would be an important step in proving Conjecture 4.1 via the polynomial partitioning method.

Remark 1.6. The results of this paper were announced in the talk [Som14] at the IPAM workshop "Tools from algebraic geometry". Shortly afterwards, a proof by Fox, Pach, Suk, Sheffer and Zahl of a weaker version of Conjecture 4.1 with an extra factor $m^{\varepsilon}$ was announced in Sheffer's blog [She14] and eventually appeared in $\left[\mathrm{FPS}^{+} 14\right]$.

Acknowledgments. We thank Zuzana Safernová/Patáková, Micha Sharir, Noam Solomon and Joshua Zahl for useful discussions and pointers to the literature. We also thank the anonymous referees for their remarks and corrections, which have significantly improved this paper.

Part of this work was done while the authors met at the Institute for Pure and Applied Mathematics (IPAM) during the Spring 2014 research program "Algebraic Techniques for Combinatorial and Computational Geometry".

\section{Preliminaries on Hilbert functions and Semi-Algebraic GeOmetry}

Throughout this paper, we denote by $\mathbb{N}$ the set of nonnegative integers. Bold letters denote finite sets or sequences of objects, where the type and number should be clear from the context: for instance, $\boldsymbol{x}$ might denote the group of variables $\left\{x_{1}, \ldots, x_{d}\right\}$ so that $\mathbb{R}[\boldsymbol{x}]$ denotes the polynomial ring $\mathbb{R}\left[x_{1}, \ldots, x_{d}\right]$.

Given functions $f, g: \mathbb{N} \rightarrow \mathbb{N}$, the Landau symbol $f=O(g)$ means that there exists $c \geq 0$ such that $f(l) \leq c g(l)$ for all $l \in \mathbb{N}$. If we want to emphasize the dependence of the constant $c$ on parameters, say $d$ and $k$, we will write $f=O_{d, k}(g)$.

2.1. Hilbert functions and degree of definition of varieties. Let $\mathbb{P}^{d}(\mathbb{C})$ denote the $d$-dimensional projective space over the complex numbers. For an equidimensional variety $X \subset \mathbb{P}^{d}(\mathbb{C})$, we denote by $\operatorname{dim}(X)$ and $\operatorname{deg}(X)$ its dimension and degree, respectively. Recall that the degree of $X$ is classically defined as the number of points in the intersection of $X$ with a generic linear subspace $H$ of dimension $d-\operatorname{dim}(X)$.

When $X$ is a hypersurface, this variety is defined by a single squarefree homogeneous polynomial $g \in \mathbb{C}\left[z_{0}, \ldots, z_{d}\right]$, unique up a scalar factor, and we have $\operatorname{deg}(X)=\operatorname{deg}(g)$. In the other extreme, when $\operatorname{dim}(X)=0$, we have $\operatorname{deg}(X)=\# X$.

A basic property of the notion of degree of varieties is its behavior with respect to intersections. In particular, it verifies the following version of Bézout's inequality [Ful84, Example 8.4.6]: let $X_{i} \subset \mathbb{P}^{d}(\mathbb{C}), i=1, \ldots t$, be equidimensional varieties 
and $Z_{j}, j=0, \ldots, l$, the irreducible components of the intersection $\bigcap_{j=1}^{t} X_{j}$. Then

$$
\sum_{j=0}^{l} \operatorname{deg}\left(Z_{j}\right) \leq \prod_{i=1}^{t} \operatorname{deg}\left(X_{i}\right) .
$$

In particular, if $g_{1}, \ldots, g_{d} \in \mathbb{C}\left[z_{0}, \ldots, z_{d}\right]$ is a family of homogeneous polynomials whose zero set in $\mathbb{P}^{d}(\mathbb{C})$ is finite, then the cardinality of this zero set is bounded by $\prod_{i=1}^{d} \operatorname{deg}\left(g_{i}\right)$.

Definition 2.1. Let $X \subset \mathbb{P}^{d}(\mathbb{C})$ be an irreducible variety and $\delta \geq 1$. We say that $X$ is partially defined at degree $\delta$ if there are homogeneous polynomials $g_{1}, \ldots, g_{t} \in$ $\mathbb{C}\left[z_{0}, \ldots, z_{d}\right]$ of degree bounded by $\delta$ such that $X$ is an irreducible component of the zero set in $\mathbb{P}^{d}(\mathbb{C})$ of these polynomials. Equivalently, there is an open subset $U \subset \mathbb{P}^{d}(\mathbb{C})$ such that $X \cap U \neq \emptyset$ and the zero set in $U$ of $g_{1}, \ldots, g_{t}$ agrees with $X \cap U$.

We denote by $\delta(X)$ the degree of partial definition of $X$, defined as the minimal integer $\delta \geq 1$ such that $X$ is partially defined at degree $\delta$.

The degree of a variety and its degree of partial definition are related by the following inequalities.

Lemma 2.2. Let $X \subset \mathbb{P}^{d}(\mathbb{C})$ be an irreducible variety. Then

$$
\delta(X) \leq \operatorname{deg}(X) \leq \delta(X)^{d-\operatorname{dim}(X)} .
$$

Proof. We first prove the left inequality. Set $e=\operatorname{dim}(X)$ and identify the projective space $\mathbb{P}^{e+1}(\mathbb{C})$ with the linear subspace of $\mathbb{P}^{d}(\mathbb{C})$ defined by the equations $z_{e+2}=$ $\cdots=z_{d}=0$. Let $L \subset \mathbb{P}^{d}(\mathbb{C})$ be a generic linear subspace of dimension $d-e-2$. By making a linear change in coordinates which keeps $z_{e+2}, \ldots, z_{d}$ unchanged, and changes only the coordinates $z_{0}, \cdots, z_{e+1}$, we can assume that $L$ is defined by the equations $z_{0}=\cdots=z_{e+1}=0$. Now consider the projection

$$
\pi_{L}: \mathbb{P}^{d}(\mathbb{C}) \backslash L \longrightarrow \mathbb{P}^{e+1}(\mathbb{C})
$$

defined, for a point $p \in \mathbb{P}^{d}(\mathbb{C}) \backslash L$, by setting $\pi_{L}(p)$ as the unique point in the intersection of $\mathbb{P}^{e+1}(\mathbb{C})$ with the linear subspace generated by $L$ and $p$. In other words $\pi_{L}\left(\left(z_{0}: \cdots: z_{d}\right)\right)=\left(z_{0}: \cdots: z_{e+1}: 0: \cdots: 0\right)$ for $\left(z_{0}: \cdots: z_{d}\right) \notin L$.

Then $\overline{\pi_{L}(X)}$, the closure of the image of $X$ under this map, is a hypersurface of $\mathbb{P}^{e+1}(\mathbb{C})$ of the same degree as $X$. This hypersurface is defined by a homogeneous polynomial $f_{L} \in \mathbb{C}\left[z_{0}, \ldots, z_{e+1}\right]$ with

$$
\operatorname{deg}\left(f_{L}\right)=\operatorname{deg}\left(\overline{\pi_{L}(X)}\right)=\operatorname{deg}(X) .
$$

Then the polynomial $f_{L}$ considered as an element of the ring $\mathbb{C}\left[z_{0}, \ldots, z_{d}\right]$ is a homogeneous polynomial of degree $\operatorname{deg}(X)$ defining a hypersurface of $\mathbb{P}^{d}(\mathbb{C})$ which contains $X$. By choosing sufficiently many linear subspaces $L$ as above, one can construct a family of homogeneous polynomials of degree $\operatorname{deg}(X)$ defining the variety $X$. Hence $\delta(X) \leq \operatorname{deg}(X)$, as stated.

For the right inequality, let $g_{1}, \ldots, g_{t} \in \mathbb{C}\left[z_{0}, \ldots, z_{d}\right]$ be a family of homogeneous polynomials of degree $\leq \delta(X)$ having $X$ as an irreducible component of its zero set. By taking generic linear combinations, we can suppose that $t=d-e$. Bézout's inequality (2.1) then implies that $\operatorname{deg}(X) \leq \delta(X)^{d-e}$, proving the inequality. 
Remark 2.3. The degree of partial definition of a variety can by much smaller than its degree. An example is provided by the Grassmannian $\operatorname{Gr}(1, n)$, the space parametrizing lines in $\mathbb{P}^{n}(\mathbb{C})$, included in the projective space $\mathbb{P}\left(\bigwedge^{2} \mathbb{C}^{n+1}\right)$ via the Plücker embedding.

The degree of this Grassmannian is

$$
\operatorname{deg}(\operatorname{Gr}(1, n))=\frac{1}{n-1}\left(\begin{array}{c}
2 n-2 \\
n
\end{array}\right)
$$

which clearly grows with $n$. On the other hand, this variety is cut out by certain quadratic equations, called the Plücker relations. Hence,

$$
\delta(\operatorname{Gr}(1, n))=2 .
$$

For irreducible varieties of codimension 2, we have the following sharpening of the second inequality in Lemma 2.2.

Lemma 2.4. Let $X \subset \mathbb{P}^{d}(\mathbb{C})$ be an irreducible variety of codimension 2. Let $\delta_{1} \geq 1$ be the minimal degree of a hypersurface of $\mathbb{P}^{d}(\mathbb{C})$ containing $X$. Then

$$
\operatorname{deg}(X) \leq \delta_{1} \delta(X)
$$

Proof. Let $f$ be a homogeneous polynomial of degree $\delta_{1} \geq 1$ vanishing on $X$. By the minimality assumption, this polynomial must be irreducible. Since $X$ is of codimension 2 , there are two homogeneous poynomials $g_{1}, g_{2}$ of degree $\leq \delta(X)$, having $X$ as an irreducible component of its zero set. By clearing common factors, we can also assume that one of these polynomials are coprime. Hence, at least one of these these polynomials (say $g_{1}$ ) is not divisible by $f$. Thus $X$ is also an irreducible component of the zero set of $f$ and $g_{1}$. The lemma then follows from Bézout's inequality (2.1).

Given a homogeneous ideal $I \subset \mathbb{C}\left[z_{0}, \ldots, z_{d}\right]$, the quotient $\mathbb{C}\left[z_{0}, \ldots, z_{d}\right] / I$ is a graded $\mathbb{C}$-algebra. The Hilbert function of $I$ is the function $\mathrm{H}_{I}: \mathbb{N} \rightarrow \mathbb{N}$ given, for $\ell \in \mathbb{N}$, by the dimension of the $\ell$-th graded piece of this quotient, that is

$$
\mathrm{H}_{I}(\ell)=\operatorname{dim}_{\mathbb{C}}\left(\mathbb{C}\left[z_{0}, \ldots, z_{d}\right] / I\right)_{\ell} .
$$

By Hilbert's theorem, there is a polynomial $\mathrm{P}_{I} \in \mathbb{Q}[t]$ and an integer $\ell_{0} \in \mathbb{N}$ with

$$
\mathrm{H}_{I}(\ell)=\mathrm{P}_{I}(\ell) \quad \text { for } \ell \geq \ell_{0} \text {. }
$$

For an equidimensional variety $X \subset \mathbb{P}^{d}(\mathbb{C})$, we denote by $I(X) \subset \mathbb{C}\left[z_{0}, \ldots, z_{d}\right]$ its defining ideal. Then $\mathrm{P}_{I(X)}$ is a polynomial of degree $\operatorname{dim}(X)$ and leading coefficient equal to the quotient $\operatorname{deg}(X) / \operatorname{dim}(X)$ !.

In Theorem 2.5 below, we collect the upper and lower bounds for Hilbert functions that we will use later on. Because of our applications, we restrict to ideals coming from irreducible projective varieties, although these bounds are valid in greater generality. Recall that binomial coefficients are defined, for $i, n \in \mathbb{Z}$, by

$$
\left(\begin{array}{c}
n \\
i
\end{array}\right)= \begin{cases}\frac{n !}{i !(n-i) !} & \text { if } 0 \leq i \leq n, \\
0 & \text { otherwise. }\end{cases}
$$

Theorem 2.5. Let $X \subset \mathbb{P}^{d}(\mathbb{C})$ be an irreducible variety of dimension $e \geq 0$.

(a) For $\ell \geq 0$,

$$
\mathrm{H}_{I(X)}(\ell) \leq \operatorname{deg}(X)\left(\begin{array}{c}
\ell+e \\
e
\end{array}\right) .
$$


(b) For $\ell \geq(d-e)(\delta(X)-1)+1$,

$$
\mathrm{H}_{I(X)}(\ell) \geq \operatorname{deg}(X)\left(\begin{array}{c}
\ell-(d-e)(\delta(X)-1)+e \\
e
\end{array}\right) .
$$

Proof. The upper bound in (a) is [Cha89, Théorème on page 306] applied to the base field $\mathbb{C}$ and the ideal $I(X)$. Similar bounds can also be derived from [Nes84] or [Som14, Proposition 2.11].

The upper bound in (b) is a particular case of [CP99, Corollaire 3]. Indeed, let $g_{1}, \ldots, g_{t} \in \mathbb{C}\left[z_{0}, \ldots, z_{d}\right]$ be a family of homogeneous polynomials of degree $\leq \delta(X)$ having $X$ as an irreducible component of its zero set. By taking generic linear combinations, we can suppose without loss of generality that $t=d-e$. Consider the ideals $I=\left(g_{1}, \ldots, g_{d-e}\right)$ and $J=I(X)$. Following the notation in page 476 of loc. cit., the ideal $I^{\langle d-e\rangle}$ is defined as the intersection of the isolated primary ideals of $I$ of codimension $d-e$. Hence $J \subset I^{\langle d-e\rangle}$. We can then apply [CP99, Corollaire 3] to these ideals. In the notation of this result, $m=d-e$, $d_{i}=\operatorname{deg}\left(g_{i}\right)$ for $i=1, \ldots, d-e$, and $r=d-e$. This result then implies that, for $\ell \geq \sum_{i=1}^{d-e} \operatorname{deg}\left(g_{i}\right)-(d-e)$

$$
\mathrm{H}_{I(X)}(\ell) \geq \operatorname{deg}(X)\left(\begin{array}{c}
\ell+d-\sum_{i=1}^{d-e} \operatorname{deg}\left(g_{i}\right) \\
e
\end{array}\right),
$$

which gives the lower bound in (b).

The following result is a consequence of Theorem 2.5(a), and appears as a particular case of [Cha89, Corollaire 3]. We include its proof, for the convenience of the interested reader.

Proposition 2.6. Let $X \subset \mathbb{P}^{d}(\mathbb{C})$ be an irreducible variety of codimension 2. Then there are coprime polynomials $f_{1}, f_{2} \in I(X)$ such that

$$
\operatorname{deg}\left(f_{1}\right) \operatorname{deg}\left(f_{2}\right) \leq d(d-1) \operatorname{deg}(X) .
$$

Proof. Set $D=\operatorname{deg}(X)$. By Theorem 2.5(a), for $\ell \geq 0$,

$$
\mathrm{H}_{I(X)}(\ell) \leq D\left(\begin{array}{c}
\ell+d-2 \\
d-2
\end{array}\right) \text {. }
$$

We have $\mathrm{H}_{\{0\}}(\ell)=\operatorname{dim}_{\mathbb{C}} \mathbb{C}[\boldsymbol{z}]_{\ell}=\left(\begin{array}{c}\ell+d \\ d\end{array}\right)$. This implies that, for $\ell_{1}=\left\lfloor(d(d-1) D)^{1 / 2}\right\rfloor$,

$$
\mathrm{H}_{I(X)}\left(\ell_{1}\right)<\mathrm{H}_{\{0\}}\left(\ell_{1}\right) .
$$

Hence, there is a homogeneous polynomial $f_{1} \in I(X) \backslash\{0\}$ with $\operatorname{deg}\left(f_{1}\right) \leq \ell_{1}$. We take $f_{1}$ of minimal degree. Since the variety $X$ is irreducible, this polynomial has to be irreducible too.

By the exact sequence

$$
0 \longrightarrow \mathbb{C}[\boldsymbol{z}] \stackrel{\times f_{1}}{\longrightarrow} \mathbb{C}[\boldsymbol{z}] \longrightarrow \mathbb{C}[\boldsymbol{z}] /\left(f_{1}\right) \longrightarrow 0,
$$

the Hilbert function of the principal ideal $\left(f_{1}\right)$ is given by

$$
\begin{aligned}
\mathrm{H}_{\left(f_{1}\right)}(\ell)=\operatorname{dim}_{\mathbb{C}} \mathbb{C}[\boldsymbol{z}]_{\ell} & -\operatorname{dim}_{\mathbb{C}} \mathbb{C}[\boldsymbol{z}]_{\ell-\ell_{1}} \\
= & \left(\begin{array}{c}
\ell+d \\
d
\end{array}\right)-\left(\begin{array}{c}
\ell-\ell_{1}+d \\
d
\end{array}\right)=\sum_{j=0}^{\ell_{1}-1}\left(\begin{array}{c}
\ell-j+d-1 \\
d-1
\end{array}\right) .
\end{aligned}
$$


Using this, one can verify that, for $\ell_{2}=\max \left\{\left\lfloor(d(d-1) D)^{1 / 2}\right\rfloor,\left\lfloor d(d-1) D / \ell_{1}\right\rfloor\right\}$,

$$
\mathrm{H}_{I(X)}\left(\ell_{2}\right)<\mathrm{H}_{\left(f_{1}\right)}\left(\ell_{2}\right) \text {. }
$$

Hence, there is a homogeneous polynomial $f_{2} \in I(X) \backslash\left(f_{1}\right)$ with $\operatorname{deg}\left(f_{2}\right) \leq \ell_{2}$. Hence, the polynomials $f_{1}, f_{2}$ are coprime and satisfy

$$
\operatorname{deg}\left(f_{1}\right) \operatorname{deg}\left(f_{2}\right) \leq \ell_{1} \ell_{2} \leq d(d-1) D,
$$

as stated.

The next result gives a lower bound for the Hilbert function of the ideal of a variety $X$ of codimension two. For $\ell \geq 0$, it exhibits three different behaviors, depending on the codimension of the zero set of the graded part $I(X)_{\ell}$.

Proposition 2.7. There is a constant $c=c(d)>0$ with the following property. Let $X \subset \mathbb{P}^{d}(\mathbb{C})$ be an irreducible variety of codimension 2. Let $\delta_{1} \geq 1$ be the minimal degree of a hypersurface of $\mathbb{P}^{d}(\mathbb{C})$ containing $X$ and set $\delta_{2}=\delta(X)$. Then

$$
\mathrm{H}_{I(X)}(\ell) \geq \begin{cases}c(\ell+1)^{d}+1 & \text { if } 1 \leq \ell \leq \delta_{1}-1, \\ c \delta_{1}(\ell+1)^{d-1}+1 & \text { if } \delta_{1} \leq \ell \leq \delta_{2}-1, \\ c \delta_{1} \delta_{2}(\ell+1)^{d-2}+1 & \text { if } \delta_{2} \leq \ell .\end{cases}
$$

Proof. We have $\delta_{1}=\min \left\{\ell \geq 0 \mid I(X)_{\ell} \neq\{0\}\right\}$. Hence, for $1 \leq \ell \leq \delta_{1}-1$,

$$
\mathrm{H}_{I(X)}(\ell)=\mathrm{H}_{\{0\}}(\ell)=\operatorname{dim}_{\mathbb{C}} \mathbb{C}[\boldsymbol{z}]_{\ell}=\left(\begin{array}{c}
\ell+d \\
d
\end{array}\right) .
$$

Thus

$$
\mathrm{H}_{I(X)}(\ell) \geq c_{1}(\ell+1)^{d}+1
$$

for a suitable constant $c_{1}>0$ depending only on $d$, giving the first lower bound.

Let $f_{1}$ be a nonzero polynomial in $I(X)$ of degree $\delta_{1}$. By the minimality property of $\delta_{1}$, this polynomial must be irreducible. We have $\delta_{2}=\min \left\{\ell \geq \delta_{1} \mid I(X)_{\ell} \neq\right.$ $\left.\left(f_{1}\right)_{\ell}\right\}$. Hence, for $\delta_{1} \leq \ell \leq \delta_{2}-1$,

$$
\mathrm{H}_{I(X)}(\ell)=\mathrm{H}_{\left(f_{1}\right)}(\ell)=\sum_{j=0}^{\delta_{1}-1}\left(\begin{array}{c}
\ell-j+d-1 \\
d-1
\end{array}\right),
$$

where the second equality comes from (2.3). It follows that, for $\delta_{1} \leq \ell \leq \delta_{2}-1$,

$$
\mathrm{H}_{I(X)}(\ell) \geq \frac{\delta_{1}}{2(d-1) !}\left(\ell-\frac{\delta_{1}}{2}\right)^{d-1}+1 \geq c_{2} \delta_{1}(\ell+1)^{d-1}+1
$$

for another constant $c_{2}=c_{2}(d)>0$.

Finally, we consider the case when $\ell \geq \delta_{2}$. When $\ell \leq 2\left(\delta_{2}-1\right)$, we deduce from (2.5) that

$$
\mathrm{H}_{I(X)}(\ell) \geq \mathrm{H}_{I(X)}\left(\delta_{2}-1\right) \geq c_{2} \delta_{1} \delta_{2}^{d-1}+1 \geq c_{3} \delta_{1} \delta_{2}(\ell+1)^{d-2}+1 .
$$

Proposition 2.6 implies that $d(d-1) \operatorname{deg}(X) \geq \delta_{1} \delta_{2}$. Hence, for $\ell \geq 2\left(\delta_{2}-1\right)+1$, Theorem 2.5(b) implies that

$$
\mathrm{H}_{I(X)}(\ell) \geq \operatorname{deg}(X)\left(\begin{array}{c}
\ell-2\left(\delta_{2}-1\right)+d-2 \\
d-2
\end{array}\right) \geq c_{4} \delta_{1} \delta_{2}(\ell+1)^{d-2}+1 .
$$

The result follows from (2.4), (2.5), (2.6) and (2.7) by taking $c=\min _{i} c_{i}$. 
2.2. Connected components of semi-algebraic sets. As explained in the introduction, the polynomial partitioning method has to be coupled with bounds for the number of connected components of semi-algebraic sets. When partitioning the Euclidean space $\mathbb{R}^{d}$, the appropriate bound follows from the Oleĭnik-Petrovskiǔ-MilnorThom's bounds for the Betti numbers of a semi-algebraic set [PO49, Mil64, Tho65]: with notation as in Theorem 1.1, the number of connected components of $\mathbb{R}^{d} \backslash V(g)$ is bounded by $\ell(2 \ell-1)^{d-1}=O\left(\ell^{d}\right)$.

In our situation, we will need the Barone-Basu bound for the number of connected components, with a refined dependence on the degrees of the polynomials [BB12, BB13]. We recall a simplified version of this result in Theorem 2.8 below.

Given $f_{1}, \ldots, f_{e} \in \mathbb{R}\left[x_{1}, \ldots, x_{d}\right]$, we denote by $V\left(f_{1}, \ldots, f_{e}\right)$ its zero set in $\mathbb{C}^{d}$. For a variety $X \subset \mathbb{C}^{d}$, we denote by $X(\mathbb{R})=X \cap \mathbb{R}^{d}$ its set of real points. For a semi-algebraic subset $S \subset \mathbb{R}^{d}$, we denote by $\operatorname{cc}(S)$ the set of connected components of $S$. The 0 -th Betti number $\mathrm{b}_{0}(S)$ coincides with the cardinality of the $\operatorname{set} \operatorname{cc}(S)$.

Theorem 2.8. There is a constant $c=c(d)$ with the following property. Let $f_{1}, \ldots, f_{e}, g \in \mathbb{R}\left[x_{1}, \ldots, x_{d}\right]$ with $\operatorname{deg}\left(f_{1}\right) \leq \cdots \leq \operatorname{deg}\left(f_{e}\right) \leq \operatorname{deg}(g)$ such that $\operatorname{dim}\left(V\left(f_{1}, \ldots, f_{i}\right)\right)=d-i$ for $i=1, \ldots, e$. Then both

$$
\mathrm{b}_{0}\left(V\left(f_{1}, \ldots, f_{e}\right)(\mathbb{R}) \backslash V(g)\right) \quad \text { and } \quad \mathrm{b}_{0}\left(V\left(f_{1}, \ldots, f_{e}, g\right)(\mathbb{R})\right)
$$

are bounded by $c \operatorname{deg}\left(f_{1}\right) \ldots \operatorname{deg}\left(f_{e}\right) \operatorname{deg}(g)^{d-e}$.

Proof. The semi-algebraic set $V\left(f_{1}, \ldots, f_{e}\right)(\mathbb{R}) \backslash V(g)$ is the union of the realization of the sign conditions \pm 1 of $g$ on $V\left(f_{1}, \ldots, f_{e}\right)(\mathbb{R})$. Similarly, $V\left(f_{1}, \ldots, f_{e}, g\right)(\mathbb{R})$ is the realization of the sign condition 0 of $g$ on the same real algebraic variety.

The result follows from [BB13, Theorem 4] and the fact that $\operatorname{dim}\left(V\left(f_{1}, \ldots, f_{i}\right)\right)$ bounds from above the dimension of the semi-algebraic set $V\left(f_{1}, \ldots, f_{i}\right)(\mathbb{R})$, see Remark 1.10 in loc. cit.

We will also need the technical result below. Given $p \in \mathbb{R}^{d}$ and $r>0$, we denote by $\mathrm{B}(p, r)$ the open ball in $\mathbb{R}^{d}$ with center $p$ and radius $r$. Given a variety $W \subset \mathbb{C}^{d}$ and a hypersurface $H \subset \mathbb{C}^{d}$, we denote by $B(W, H)$ the subset of $W(\mathbb{R})$ of points $p \in W(\mathbb{R})$ having an open neighborhood, in the Euclidean topology of $W(\mathbb{R})$, contained in $H$. We also set $G(W, H)=W(\mathbb{R}) \backslash B(W, H)$.

Proposition 2.9. Let $W \subset \mathbb{C}^{4}$ be a variety and $H, K \subset \mathbb{C}^{4}$ two hypersurfaces. Let $b \in \mathbb{R}\left[x_{1}, x_{2}, x_{3}, x_{4}\right]$ be a polynomial defining $H$. Then there exists $\varepsilon_{0}>0$ such that, for all $0<\varepsilon \leq \varepsilon_{0}$ and any variety $\widetilde{W} \subset \mathbb{C}^{4}$ containing $W$, the number of connected components $C$ of $\mathbb{R}^{4} \backslash K$ such that $C \cap G(W, H) \cap H \neq \emptyset$ is bounded by

$$
\mathrm{b}_{0}\left(\left(\widetilde{W}(\mathbb{R}) \cap V\left(b^{2}-\varepsilon\right)\right) \backslash K\right) .
$$

Proof. Consider the set of connected components

$$
\mathcal{C}=\left\{C \in \operatorname{cc}\left(\mathbb{R}^{4} \backslash K\right) \mid C \cap G(W, H) \cap H \neq \emptyset\right\} .
$$

For each $C \in \mathcal{C}$ choose a point $p_{C} \in C \cap G(W, H) \cap H$. Since $\mathcal{C}$ is a finite set, the set of points $\left\{p_{C}\right\}_{C \in \mathcal{C}}$ is finite. For each $C \in \mathcal{C}$ and $r>0$, consider also the semi-algebraic set given by

$$
U_{r}\left(p_{C}\right)=\mathrm{B}\left(p_{C}, r\right) \cap(W(\mathbb{R}) \backslash K) .
$$

By the definition of $G(W, H)$, the set $U_{r}\left(p_{c}\right)$ is not contained in $H$. Semi-algebraic sets are locally contractible because of their local conical structure, see for instance 
[BPR06, Theorem 5.48]. Hence, there exists $r_{C}>0$ such that, for all $0<r \leq r_{C}$, the set $U_{r}\left(p_{C}\right)$ is contractible and, in particular, connected. Set $r_{0}=\min _{C} r_{C}$.

Choose also $q_{C} \in U_{r_{0}}\left(p_{C}\right) \backslash H$ and a semi-algebraic path $\gamma_{C}:[0,1] \rightarrow U_{r_{0}}\left(p_{C}\right)$ with $\gamma(0)=p_{C}$ and $\gamma(1)=q_{C}$. We have that $b^{2}\left(q_{C}\right)>0$ because $q_{C} \notin H$. We set $\varepsilon_{0}=\min _{C} b^{2}\left(q_{C}\right)$.

By the intermediate value theorem, for all $C \in \mathcal{C}$ and $0<\varepsilon \leq \varepsilon_{0}$, there exists $0<t_{C} \leq 1$ such that $b^{2}\left(z_{C}\right)=\varepsilon$ with $z_{C}=\gamma_{C}\left(t_{C}\right)$. By construction,

$$
z_{C} \in\left(W(\mathbb{R}) \cap V\left(b^{2}-\varepsilon\right)\right) \backslash K \subset\left(\widetilde{W}(\mathbb{R}) \cap V\left(b^{2}-\varepsilon\right)\right) \backslash K .
$$

Moreover, $z_{C} \in C$ because this point is connected by a path to $p_{C}$. For $C, C^{\prime} \in \mathcal{C}$ with $C \neq C^{\prime}$, the points $z_{C}$ and $z_{C^{\prime}}$ belong to distinct connected components of $\widetilde{W}(\mathbb{R}) \cap V\left(b^{2}-\varepsilon\right) \backslash K$. Hence, the map $C \mapsto z_{C}$ induces an injection between the set of connected components $\mathcal{C}$ and $\operatorname{cc}\left(\widetilde{W}(\mathbb{R}) \cap V\left(b^{2}-\varepsilon\right)\right) \backslash K$, which proves the proposition.

In connection with the application of the polynomial partitioning theorem to incidence problems in higher dimensions, we propose the following conjectural bound for the number of connected components of a semi-algebraic set in terms of the degree of the variety instead of the Bézout number of a set of defining equations.

Conjecture 2.10. Let $X \subset \mathbb{C}^{d}$ be an irreducible variety and $g \in \mathbb{R}\left[x_{1}, \ldots, x_{d}\right]$ a polynomial of degree $\ell \geq \delta(X)$. Then there exists a variety $Y \subset \mathbb{C}^{d}$ containing $X$ as an irreducible component such that

$$
\mathrm{b}_{0}(Y(\mathbb{R}) \backslash V(g)) \quad \text { and } \quad \mathrm{b}_{0}(Y(\mathbb{R}) \cap V(g))
$$

are bounded by $O_{d}\left(\operatorname{deg}(X) \ell^{\operatorname{dim}(X)}\right)$.

When $X$ is an irreducible variety of codimension 2, this statement follows easily from Proposition 2.6 and Theorem 2.8. In this case, the variety $Y$ is given, in the notation of Proposition 2.6, by the zero set of $f_{1}$ and $f_{2}$.

\section{Partitioning Finite SETS ON VARIETies}

Given a set of points $\mathcal{P} \subset \mathbb{R}^{d}$ and a set of polynomials $\mathcal{G} \subset \mathbb{R}\left[x_{1}, \ldots, x_{d}\right]$, for each choice of signs $\gamma \in\{ \pm 1\}^{\mathcal{G}}$ we put

$$
\mathcal{P}(\gamma)=\left\{p \in \mathcal{P} \mid \gamma_{g} g(p)>0 \text { for all } g \in \mathcal{G}\right\},
$$

If the set of polynomials $\mathcal{G}$ is clear from the context, then we say that $\mathcal{P}(\gamma)$ is realized by $\gamma$.

Given $g \in \mathbb{R}\left[x_{1}, \ldots, x_{d}\right] \backslash\{0\}$, we denote by $\operatorname{irr}(g) \subset \mathbb{R}\left[x_{1}, \ldots, x_{d}\right]$ a complete and irredundant set of irreducible factors of $g$. These irreducible factors are unique up to scalars in $\mathbb{R}^{\times}$. To fix their indeterminacy, we choose them to be monic with respect to some fixed monomial order on $\mathbb{R}\left[x_{1}, \ldots, x_{d}\right]$. With this convention, the set $\operatorname{irr}(g)$ is uniquely defined and

$$
g=\lambda \prod_{q \in \operatorname{irr}(g)} q^{e_{q}}
$$

with $\lambda \in \mathbb{R}^{\times}$and $e_{q} \in \mathbb{N}$.

For $\ell \geq 0$, we denote by $\mathbb{R}\left[x_{1}, \ldots, x_{d}\right]_{\leq \ell}$ the linear subspace of $\mathbb{R}\left[x_{1}, \ldots, x_{d}\right]$ of polynomials of degree bounded by $\ell$. Recall that, for a variety $X \subset \mathbb{C}^{d}$, we denote by $X(\mathbb{R})=X \cap \mathbb{R}^{d}$ its set of real points. 
We state and prove our polynomial partitioning theorem in terms of sign conditions. For convenience, we state it for varieties of codimension at most two, even though we prove it only when the codimension is two. The cases when the codimension is smaller are simpler and can be proven as in [GK10, Zah13, KMSS12].

Theorem 3.1. Let $X \subset \mathbb{C}^{d}$ be an irreducible variety of codimension at most two, $\mathcal{P} \subset X(\mathbb{R})$ a finite subset and $\ell \geq 6 d \delta(X)$. Then there exists $g \in \mathbb{R}\left[x_{1}, \ldots, x_{d}\right]_{\leq \ell}$ with $\operatorname{dim}(X \cap V(g))=\operatorname{dim}(X)-1$ such that, for each $\gamma \in\{ \pm 1\}^{\operatorname{irr}(g)}$,

$$
\operatorname{card}(\mathcal{P}(\gamma))=O_{d}\left(\frac{\operatorname{card}(\mathcal{P})}{\operatorname{deg}(X) \ell^{\operatorname{dim}(X)}}\right) .
$$

Remark 3.2. Let $S \subset \mathbb{R}^{d}$ be an arbitrary subset. For each connected component $C$ of $S \backslash V(g)$, the set $\mathcal{P} \cap C$ is contained in a set of the form $\mathcal{P}(\gamma)$ with $\gamma \in\{ \pm 1\}^{\operatorname{irr}(g)}$. Hence, Theorem 1.2 in the introduction follows from Theorem 3.1 above by choosing $S=\mathbb{R}^{d}$.

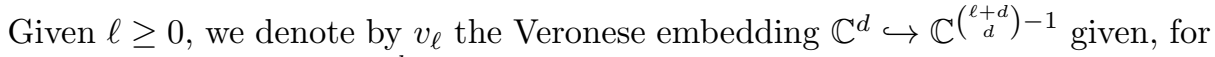
a point $\boldsymbol{p}=\left(p_{1}, \ldots, p_{d}\right) \in \mathbb{C}^{d}$, by

$$
v_{\ell}(\boldsymbol{p})=\left(\boldsymbol{p}^{\boldsymbol{a}}\right)_{\boldsymbol{a}}
$$

where $\boldsymbol{a}=\left(a_{1}, \ldots, a_{d}\right) \in \mathbb{N}^{d}$ runs over all nonzero vectors of length $|\boldsymbol{a}|=\sum_{i} a_{i}$ bounded by $\ell$, and where $\boldsymbol{p}^{a}$ denotes the monomial $p_{1}^{a_{1}} \ldots p_{d}^{a_{d}}$. We also denote by $\iota$ the standard inclusion $\mathbb{C}^{d} \rightarrow \mathbb{P}^{d}(\mathbb{C})$ given by

$$
\iota(\boldsymbol{p})=\left(1: p_{1}: \cdots: p_{d}\right) .
$$

For a subset $E \subset \mathbb{R}^{d}$, we denote by aff $(E)$ the smallest affine subspace (or flat) of $\mathbb{R}^{d}$ containing $E$. We also denote by $I(\iota(E)) \subset \mathbb{C}\left[z_{0}, \ldots, z_{d}\right]$ the homogeneous ideal of polynomials vanishing identically on the subset $\iota(E) \subset \mathbb{P}^{d}(\mathbb{C})$.

Lemma 3.3. With notation as above, let $E \subset \mathbb{R}^{d}$ be a subset and $\ell \geq 0$. Then

$$
\operatorname{dim}_{\mathbb{R}}\left(\operatorname{aff}\left(v_{\ell}(E)\right)\right)=\mathrm{H}_{I(\iota(E))}(\ell)-1 .
$$

Proof. The ideal $I(\iota(E))$ is generated over $\mathbb{R}[\boldsymbol{z}]$, because it is defined by the vanishing of a set of real points. Setting $I=I(\iota(E)) \cap \mathbb{R}[\boldsymbol{z}]$, we have

$$
\mathrm{H}_{I(\iota(E))}(\ell)=\operatorname{dim}_{\mathbb{C}}(\mathbb{C}[\boldsymbol{z}] / I(\iota(E)))_{\ell}=\operatorname{dim}_{\mathbb{R}}\left(\mathbb{R}[\boldsymbol{z}]_{\ell}\right)-\operatorname{dim}_{\mathbb{R}}\left(I_{\ell}\right),
$$

where $I_{\ell}$ denotes the $\ell$-th graded part of $I$.

Consider the Euclidean space $\mathbb{R}^{(\ell+d)} d$ with coordinates indexed by the vectors of $\mathbb{N}^{d+1}$ of length equal to $\ell$, and the pairing defined by

$$
\mathbb{R}[\boldsymbol{z}]_{\ell} \times \mathbb{R}^{\left(\begin{array}{c}
\ell+d \\
d
\end{array}\right)} \longrightarrow \mathbb{R}, \quad\left(\sum_{|\boldsymbol{b}|=\ell} \alpha_{\boldsymbol{b}} \boldsymbol{z}^{\boldsymbol{b}}, \boldsymbol{w}\right) \longmapsto \sum_{\boldsymbol{b}} \alpha_{\boldsymbol{b}} \boldsymbol{w}_{\boldsymbol{b}},
$$

where $\boldsymbol{b}$ runs over all vectors of $\mathbb{N}^{d+1}$ of length $\ell$.

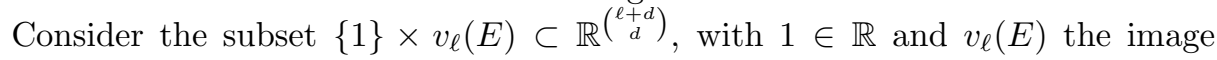
of $E$ under the Veronese embedding (3.2). The graded part $I_{\ell}$ coincides with the annihilator of this subset with respect to the pairing (3.4). Since $I_{\ell}$ is a linear subspace, it also coincides with the annihilator of the linear span in $\mathbb{R}^{\left(\begin{array}{c}\ell+d \\ d\end{array}\right)}$ of this subset. Denote by $\operatorname{lin}\left(\{1\} \times v_{\ell}(E)\right)$ this linear span, which is a linear space containing $\{1\} \times \operatorname{aff}\left(v_{\ell}(E)\right)$ as an affine hyperplane. Hence

$$
\operatorname{dim}_{\mathbb{R}}\left(\mathbb{R}[\boldsymbol{z}]_{\ell}\right)-\operatorname{dim}_{\mathbb{R}}\left(I_{\ell}\right)=\operatorname{dim}_{\mathbb{R}}\left(\operatorname{lin}\left(\{1\} \times v_{\ell}(E)\right)\right)=\operatorname{dim}_{\mathbb{R}}\left(\operatorname{aff}\left(v_{\ell}(E)\right)\right)+1 .
$$


The result then follows from (3.3) and (3.5).

Proof of Theorem 3.1. We assume that $X$ is of codimension 2. Let $\delta_{1} \geq 1$ be the minimal degree of a hypersurface of $\mathbb{P}^{d}(\mathbb{C})$ containing $X$ and set also $\delta_{2}=\delta(X)$. Let $\eta \geq \delta_{2}$ be an integer to be fixed later on.

Let $c=c(d)$ be the constant in Proposition 2.7 and set $c_{1}=\min \left\{c, 2^{-d}\right\}$. Put

$$
s_{0}=\log \left(c_{1} \delta_{1}^{d}\right), \quad s_{1}=\log \left(c_{1} \delta_{1} \delta_{2}^{d-1}\right), \quad t=\left\lfloor\log \left(c_{1} \delta_{1} \delta_{2} \eta^{d-2}\right)\right\rfloor
$$

and

$$
\ell_{i}= \begin{cases}\left\lfloor\left(c_{1}^{-1} 2^{i}\right)^{\frac{1}{d}}\right\rfloor & \text { for } 0 \leq i<s_{0}, \\ \left\lfloor\left(c_{1}^{-1} \delta_{1}^{-1} 2^{i}\right)^{\frac{1}{d-1}}\right\rfloor & \text { for } s_{0} \leq i<s_{1}, \\ \left\lfloor\left(c_{1}^{-1} \delta_{1}^{-1} \delta_{2}^{-1} 2^{i}\right)^{\frac{1}{d-2}}\right\rfloor & \text { for } s_{1} \leq i \leq t,\end{cases}
$$

where $\lfloor\cdot\rfloor$ denotes the floor function. We verify that the following conditions hold:

$$
\begin{aligned}
& \text { if } 0 \leq i<s_{0} \text { then } 1 \leq \ell_{i} \leq \delta_{1}-1, \\
& \text { if } s_{0} \leq i<s_{1} \text { then } \delta_{1} \leq \ell_{i} \leq \delta_{2}-1, \\
& \text { if } s_{1} \leq i \leq t \text { then } \delta_{2} \leq \ell_{i} \leq \eta
\end{aligned}
$$

Let $v_{\ell_{i}}$ be the Veronese map of degree $\ell_{i}$ as in (3.2) and set $A_{i} \subset \mathbb{R}^{\left(\begin{array}{c}\ell_{i}+e \\ e\end{array}\right)}-1$ for the affine hull of the image of $X(\mathbb{R})$ under $v_{\ell_{i}}$. Let $I(\iota(X(\mathbb{R})))$ be the ideal of polynomials vanishing on the image under $\iota$ of the set of real points of $X$. By Lemma 3.3,

$$
\operatorname{dim}_{\mathbb{R}}\left(A_{i}\right)=\mathrm{H}_{I(\iota(X(\mathbb{R})))}\left(\ell_{i}\right)-1 .
$$

Since $\iota(X(\mathbb{R})) \subset \iota(X)$, we have that $I(\iota(X(\mathbb{R}))) \supset I(\iota(X))$ and so

$$
\mathrm{H}_{I(\iota(X(\mathbb{R})))}\left(\ell_{i}\right) \leq \mathrm{H}_{I(\iota(X))}\left(\ell_{i}\right) .
$$

We consider first the case when (3.8) is an equality for all $i$. Since the affine variety $X$ is irreducible and partially defined at degree $\delta(X)$, the same holds for $\overline{\iota(X)}$, the Zariski closure of $\iota(X)$ in projective space. It follows from Proposition 2.7 and the conditions in (3.6) that

$$
\mathrm{H}_{I(\iota(X(\mathbb{R})))}\left(\ell_{i}\right) \geq 2^{i}+1, \quad i=0, \ldots, t .
$$

As in the Guth-Katz polynomial partitioning, we will inductively subdivide the set of points $\mathcal{P}$. We start with $\mathcal{C}_{0}=\{\mathcal{P}\}$. Having constructed $\mathcal{C}_{i}$ with at most $2^{i}$ sets, we apply the ham sandwich theorem to the image of these sets under the map $v_{\ell_{i}}$. These images lie in $A_{i}$ and, by (3.7) and (3.9), this is an affine space of dimension $\geq 2^{i}$. Hence, there is a nonzero linear form on $A_{i}$ that bisects each of these images or, equivalently, there is a polynomial $g_{i} \in \mathbb{R}\left[x_{1}, \ldots, x_{d}\right]_{\leq \ell_{i}}$ bisecting each of the sets in $\mathcal{C}_{i}$.

For each $\mathcal{Q} \in \mathcal{C}_{i}$, we put $\mathcal{Q}^{+}$and $\mathcal{Q}^{-}$for the sets of points of $\mathcal{Q}$ at which $g_{i}>0$ and $g_{i}<0$, respectively. We then put

$$
\mathcal{C}_{i+1}=\bigcup_{\mathcal{Q} \in \mathcal{C}_{i}}\left\{\mathcal{Q}^{+}, \mathcal{Q}^{-}\right\}
$$

Hence, each of the sets in $\mathcal{C}_{t}$ has cardinality bounded by $2^{-t} \operatorname{card}(\mathcal{P})$.

Set $g=\prod_{i=0}^{t} g_{i}$. To bound the degree of $g$, we write $\operatorname{deg}(g)=S_{0}+S_{1}+S_{2}$ with

$$
S_{0}=\sum_{0 \leq i<s_{0}} \ell_{i}, \quad S_{1}=\sum_{s_{0} \leq i<s_{1}} \ell_{i}, \quad S_{2}=\sum_{s_{1} \leq i \leq t} \ell_{i} .
$$


We have that

$$
S_{0} \leq \sum_{i=0}^{s_{0}-1}\left(c_{1}^{-1} 2^{i}\right)^{\frac{1}{d}} \leq c_{1}^{-\frac{1}{d}} \frac{2^{\frac{s_{0}+1}{d}}-1}{2^{\frac{1}{d}}-1} \leq \frac{2^{\frac{1}{d}}}{2^{\frac{1}{d}}-1} \delta_{1} .
$$

Similarly, one can verify that

$$
S_{1} \leq \frac{2^{\frac{1}{d-1}}}{2^{\frac{1}{d-1}}-1} \delta_{2} \quad \text { and } \quad S_{2} \leq \frac{2^{\frac{1}{d-2}}}{2^{\frac{1}{d-2}}-1} \eta .
$$

Using that $d \geq 3$ and $\delta_{1} \leq \delta_{2}$, we deduce that $\operatorname{deg}(g) \leq 4 d \delta_{2}+2 d \eta$. Finally, set

$$
\eta=\frac{\ell}{2 d}-2 \delta_{2} \text {. }
$$

Since $\ell \geq 6 d \delta_{2}$, we have that $\eta \geq \delta_{2}$ as required and $\operatorname{deg}(g) \leq \ell$, as stated.

On the other hand, the sets in $\mathcal{C}_{t}$ are realized by sign conditions given in terms of the $g_{i}$ 's. The sets realized by sign conditions on $\operatorname{irr}(g)$ have cardinality bounded by those in $\mathcal{C}_{t}$. Since $\eta \geq \frac{\ell}{6 d}$, it follows that for each $\gamma \in\{ \pm 1\}^{\operatorname{irr}(g)}$,

$$
\operatorname{card}(\mathcal{P}(\gamma)) \leq \frac{\operatorname{card}(\mathcal{P})}{2^{t}} \leq c_{2} \frac{\operatorname{card}(\mathcal{P})}{\operatorname{deg}(X) \eta^{d-2}},
$$

where the last inequality follows from Lemma 2.4, and $c_{2}$ denotes a suitable constant. This proves the statement in the case when $X$ is of codimension 2 and the inequality (3.8) are equalities for all $i$.

If the inequality (3.8) is strict for some $i$, then there is a polynomial $g_{i} \in$ $I(X(\mathbb{R})) \backslash I(X)$ of degree bounded by $\ell_{i} \leq \ell$. Hence, the hypersurface $V\left(g_{i}\right)$ cuts $X$ properly and contains its set of real points. In particular, $\mathcal{P} \subset V\left(g_{i}\right)$. It follows that $g=g_{i}$ has the appropriate degree and $\mathcal{P}(\gamma)=\emptyset$ for all $\gamma \in\{ \pm 1\}^{\operatorname{irr}(g)}$, which completes the proof for the case when $X$ is of codimension two.

The cases when the codimension of $X$ is either zero or one are simpler and can be proven as in [GK10, Zah13, KMSS12].

A previous version of this paper contained a polynomial partitioning theorem on varieties of arbitrary dimension. Whereas the proof of this result contained a gap, we still think that its statement is correct, and we propose it as a conjecture.

Conjecture 3.4. There is a constant $c=c(d)>0$ with the following property. Let $X \subset \mathbb{C}^{d}$ be an irreducible variety of dimension $e, \mathcal{P} \subset X(\mathbb{R})$ a finite subset and $\ell \geq c \delta(X)$. Then there exists $g \in \mathbb{R}\left[x_{1}, \ldots, x_{d}\right]_{\leq \ell}$ with $\operatorname{dim}(X \cap V(g))=\operatorname{dim}(X)-1$ such that, for each $\gamma \in\{ \pm 1\}^{\operatorname{irr}(g)}$,

$$
\operatorname{card}(\mathcal{P}(\gamma)) \leq c \frac{\operatorname{card}(\mathcal{P})}{\operatorname{deg}(X) \ell^{e}}
$$

\section{Point-hypersurface incidences}

In this section we prove Theorem 1.5. To this end, we use three levels of polynomial partitioning. This leads to a partition of the Euclidean space $\mathbb{R}^{4}$ into semialgebraic pieces of various dimensions. We bound separately the number of incidences contributed by the points of the set $\mathcal{P}$ in each piece. The contribution from each level of the partitioning is essentially the same, up to constant factors, as the claimed bound. 
Proof of Theorem 1.5. The procedure performed at each level is similar. For clarity and ease of exposition, we prefer to describe each of these level separately, even at the expense of repeating some of the arguments.

The set of incidences between $\mathcal{P}$ and $\mathcal{H}$ is the subset of $\mathcal{P} \times \mathcal{H}$ defined by

$$
\mathcal{I}(\mathcal{P}, \mathcal{H})=\{(p, H) \in \mathcal{P} \times \mathcal{H} \mid p \in H\} .
$$

Hence $I(\mathcal{P}, \mathcal{H})=\operatorname{card}(\mathcal{I}(\mathcal{P}, \mathcal{H}))$. For a subset $\mathcal{Q} \subset \mathcal{P}$, we denote by

$$
\begin{aligned}
& \mathcal{I}_{<k}(\mathcal{Q}, \mathcal{H})=\{(p, H) \in \mathcal{I}(\mathcal{Q}, \mathcal{H}) \mid \operatorname{card}(H \cap \mathcal{Q})<k\}, \\
& \mathcal{I}_{\geq k}(\mathcal{Q}, \mathcal{H})=\{(p, H) \in \mathcal{I}(\mathcal{Q}, \mathcal{H}) \mid \operatorname{card}(H \cap \mathcal{Q}) \geq k\}
\end{aligned}
$$

the set of incidences between $\mathcal{Q}$ and hypersurfaces of $\mathcal{H}$ containing at most $k-1$ points of $\mathcal{Q}$ and at least $k$ points of $\mathcal{Q}$, respectively. We also set $I_{<k}(\mathcal{Q}, \mathcal{H})=$ $\operatorname{card}\left(\mathcal{I}_{<k}(\mathcal{Q}, \mathcal{H})\right)$ and $I_{\geq k}(\mathcal{Q}, \mathcal{H})=\operatorname{card}\left(\mathcal{I}_{\geq k}(\mathcal{Q}, \mathcal{H})\right)$. Clearly,

$$
I(\mathcal{Q}, \mathcal{H})=I_{<k}(\mathcal{Q}, \mathcal{H})+I_{\geq k}(\mathcal{Q}, \mathcal{H}) .
$$

In the sequel, the dimension $d$ of the ambient space is fixed to 4 . Hence, all implicit constants in the $O$-notation depend only on the parameters $k$ and $c$ in the statement of the theorem.

First level partitioning. Let $D \geq 24$ to be fixed later on. By Theorem 3.1, there exists $f \in \mathbb{R}\left[x_{1}, x_{2}, x_{3}, x_{4}\right]_{\leq D} \backslash\{0\}$ such that, for each $\gamma \in\{ \pm 1\}^{\operatorname{irr}(f)}$,

$$
\operatorname{card}(\mathcal{P}(\gamma))=O\left(\frac{m}{D^{4}}\right),
$$

where $\mathcal{P}(\gamma)$ denotes the subset of $\mathcal{P}$ realized by the signs $\gamma$ as in (3.1). Choose a minimal subset $\Sigma_{1} \subset\{ \pm 1\}^{\operatorname{irr}(f)}$ realizing all nonempty subsets of this form.

We partition $\mathcal{P}$ into the disjoint subsets $\mathcal{P}_{0}=\mathcal{P} \cap V(f)$ and $\mathcal{P}(\gamma), \gamma \in \Sigma_{1}$. Set $m_{0}=\operatorname{card}\left(\mathcal{P}_{0}\right)$ and $m_{\gamma}=\operatorname{card}(\mathcal{P}(\gamma))$ for each $\gamma$. Clearly,

$$
m_{0}+\sum_{\gamma \in \Sigma_{1}} m_{\gamma}=m
$$

We first bound the number of incidences with hypersurfaces that contain at least $k$ points in one of the subsets $\mathcal{P}(\gamma)$. By the hypothesis (c), for each $\gamma \in \Sigma_{1}$ and each subset of $k$ points of $\mathcal{P}(\gamma)$, there are at most $c$ hypersurfaces in $\mathcal{H}$ containing these points. Hence,

$$
I_{\geq k}(\mathcal{P}(\gamma), \mathcal{H}) \leq c k\left(\begin{array}{c}
m_{\gamma} \\
k
\end{array}\right)=O\left(m_{\gamma}^{k}\right) .
$$

The cardinality of $\Sigma_{1}$ or equivalently, the number of nonempty subsets of the form $\mathcal{P}(\gamma)$, is bounded by the number of connected components of $\mathbb{R}^{4} \backslash V(f)$. By Theorem 2.8, applied with $e=0$, this number is bounded by $O\left(D^{4}\right)$. With (4.2) and (4.3), this implies that

$$
\sum_{\gamma} I_{\geq k}(\mathcal{P}(\gamma), \mathcal{H})=O\left(\sum_{\gamma}\left(\frac{m}{D^{4}}\right)^{k}\right)=O\left(m^{k} D^{4-4 k}\right) .
$$

We now bound the number of incidences with hypersurfaces that contain at most $k-1$ points in every $\mathcal{P}(\gamma)$. For each $H \in \mathcal{H}$, the number of subsets $\mathcal{P}(\gamma)$ having nonempty intersection with $H$ is bounded by $\mathrm{b}_{0}(H(\mathbb{R}) \backslash V(f))$. By Theorem 2.8, this number of connected components is bounded by $O\left(D^{3}\right)$, because the degree of $H$ is 
bounded by a constant. Hence $\sum_{\gamma} I_{<k}(\mathcal{P}(\gamma),\{H\}) \leq(k-1) \mathrm{b}_{0}(H \backslash V(f))=O\left(D^{3}\right)$. It follows that

$$
\sum_{\gamma} I_{<k}(\mathcal{P}(\gamma), \mathcal{H})=O\left(n D^{3}\right) .
$$

From (4.1), (4.4) and (4.5) we deduce that

$$
I\left(\mathcal{P} \backslash \mathcal{P}_{0}, \mathcal{H}\right)=\sum_{\gamma} I(\mathcal{P}(\gamma), \mathcal{H})=O\left(n D^{3}+m^{k} D^{4-4 k}\right) .
$$

We then set

$$
D=\max \left(24, \frac{m^{\alpha_{1}}}{n^{\beta_{1}}}\right) \quad \text { with } \alpha_{1}=\frac{k}{4 k-1} \text { and } \beta_{1}=\frac{1}{4 k-1} .
$$

If $D=24$, then $m^{\alpha_{1}} n^{-\beta_{1}} \leq 24$ and so $m^{k}=O(n)$. In this case, it follows from (4.6) that $I\left(\mathcal{P} \backslash \mathcal{P}_{0}, \mathcal{H}\right)=O\left(n+m^{k}\right)=O(n)$. Otherwise,

$$
I\left(\mathcal{P} \backslash \mathcal{P}_{0}, \mathcal{H}\right)=O\left(m^{3 \alpha_{1}} n^{1-3 \beta_{1}}\right)=O\left(m^{1-\frac{k-1}{4 k-1}} n^{1-\frac{3}{4 k-1}}\right) .
$$

In either case,

$$
I\left(\mathcal{P} \backslash \mathcal{P}_{0}, \mathcal{H}\right)=O\left(m^{1-\frac{k-1}{4 k-1}} n^{1-\frac{3}{4 k-1}}+n\right) .
$$

Second level partitioning. Let $V(f)=\bigcup_{i \in I} V_{i}$ be the decomposition of the hypersurface $V(f)$ into irreducible components. Set $D_{i}=\operatorname{deg}\left(V_{i}\right)$ for each $i \in I$. Then

$$
\sum_{i \in I} D_{i}=\operatorname{deg}(V(f)) \leq D \text {. }
$$

We choose a partition of the finite set $\mathcal{P}_{0}=\mathcal{P} \cap V(f)$ into disjoint subsets $\mathcal{Q}_{i}$, $i \in I$, by assigning each point in $\mathcal{P}_{0}$ to one of the subsets $\mathcal{Q}_{i}$ corresponding to an irreducible component $V_{i}$ it belongs to. Set $l_{i}=\operatorname{card}\left(\mathcal{Q}_{i}\right)$ for each $i \in I$. Then

$$
\sum_{i} l_{i}=m_{0}
$$

Fix $i \in I$ and let $E_{i} \geq 24 D_{i}$. By Theorem 3.1, there exists $g_{i} \in \mathbb{R}\left[x_{1}, x_{2}, x_{3}, x_{4}\right]_{\leq E_{i}}$ such that $\operatorname{dim}\left(V_{i} \cap V\left(g_{i}\right)\right)=2$ and, for each $\delta \in\{ \pm 1\}^{\operatorname{irr}\left(g_{i}\right)}$,

$$
\operatorname{card}\left(\mathcal{Q}_{i}(\delta)\right)=O\left(\frac{l_{i}}{D_{i} E_{i}^{3}}\right) .
$$

Choose a minimal subset $\Sigma_{2, i} \subset\{ \pm 1\}^{\operatorname{irr}\left(g_{i}\right)}$ realizing all nonempty subsets of the form $\mathcal{Q}_{i}(\delta)$.

Consider the surface $W_{i}=V_{i} \cap V\left(g_{i}\right)=V\left(f_{i}, g_{i}\right)$ and partition $\mathcal{Q}_{i}$ into the disjoint subsets $\mathcal{Q}_{i, 0}=\mathcal{Q}_{i} \cap W_{i}$ and $\mathcal{Q}_{i}(\delta), \delta \in \Sigma_{2, i}$. We set $l_{i, 0}=\operatorname{card}\left(\mathcal{Q}_{i, 0}\right)$ and $l_{i, \delta}=\operatorname{card}\left(\mathcal{Q}_{i}(\delta)\right)$ for each $\delta$. Clearly,

$$
l_{i, 0}+\sum_{\delta \in \Sigma_{2, i}} l_{i, \delta}=l_{i} \quad \text { and } \quad \sum_{i} l_{i, 0}=\operatorname{card}\left(\mathcal{P} \cap \bigcup_{i} W_{i}\right) .
$$

We follow the same approach as in the previous case, and we first bound the number of incidences with hypersurfaces that contain at least $k$ points in some $\mathcal{Q}_{i}(\delta)$. Similarly as in (4.3), the hypothesis (c) implies that, for each $\delta$,

$$
I_{\geq k}\left(\mathcal{Q}_{i}(\delta), \mathcal{H}\right) \leq c k\left(\begin{array}{c}
l_{i, \delta} \\
k
\end{array}\right)=O\left(l_{i, \delta}^{k}\right) .
$$


The cardinality of $\Sigma_{2, i}$ is bounded by $\mathrm{b}_{0}\left(V_{i}(\mathbb{R}) \backslash V\left(g_{i}\right)\right)$ which, by Theorem 2.8 , is bounded by $O\left(D_{i} E_{i}^{3}\right)$. With (4.11) and (4.12), this implies that

$$
\sum_{\delta} I_{\geq k}\left(\mathcal{Q}_{i}(\delta), \mathcal{H}\right)=O\left(\sum_{\delta}\left(\frac{l_{i}}{D_{i} E_{i}^{3}}\right)^{k}\right)=O\left(l_{i}^{k} D_{i}^{1-k} E_{i}^{3-3 k}\right) .
$$

We now bound the number of incidences with hypersurfaces that contain at most $k-1$ points in every $\mathcal{Q}_{i}(\delta)$. Let $H \in \mathcal{H}$ and, for the moment, suppose that $V_{i} \not \subset H$. Since $V_{i}$ is an algebraic variety over $\mathbb{C}$ with $\operatorname{dim}\left(V_{i}\right)=3$, by [Har77, Chapter I, Proposition 7.1] we have that either $H \cap V_{i}$ is empty or of dimension 2. Moreover, the degree of $H$ is bounded by a constant. The number of subsets of the form $\mathcal{Q}_{i}(\delta)$ with nonempty intersection with $H$ is bounded by $b_{0}\left(\left(H \cap V_{i}\right)(\mathbb{R}) \backslash V\left(g_{i}\right)\right)$. By Theorem 2.8, this number is bounded by $O\left(D_{i} E_{i}^{2}\right)$. If we note by $\mathcal{H}_{i}$ the set of hypersurfaces of $\mathcal{H}$ not containing $V_{i}$, then

$$
\sum_{\delta} I_{<k}\left(\mathcal{Q}_{i}(\delta), \mathcal{H}_{i}\right)=O\left(n D_{i} E_{i}^{2}\right)
$$

On the other hand, by the hypothesis (b), there are at most 3 hypersurfaces $H \in \mathcal{H}$ containing $V_{i}$, and each of them contains the $l_{i}$ points of $\mathcal{Q}_{i}$. Hence

$$
I_{<k}\left(\mathcal{Q}_{i} \backslash \mathcal{Q}_{i, 0}, \mathcal{H} \backslash \mathcal{H}_{i}\right) \leq I\left(\mathcal{Q}_{i}, \mathcal{H} \backslash \mathcal{H}_{i}\right) \leq 3 l_{i} .
$$

By (4.13) and (4.14),

$$
I\left(\mathcal{Q}_{i} \backslash \mathcal{Q}_{i, 0}, \mathcal{H}\right)=\sum_{\delta} I\left(\mathcal{Q}_{i}(\delta), \mathcal{H}\right)=O\left(n D_{i} E_{i}^{2}+l_{i}^{k} D_{i}^{1-k} E_{i}^{3-3 k}+l_{i}\right) .
$$

We set

$$
E_{i}=\max \left(24 D_{i},\left(\frac{l_{i}}{D_{i}}\right)^{\alpha_{2}} \frac{1}{n^{\beta_{2}}}\right) \quad \text { with } \alpha_{2}=\frac{k}{3 k-1} \text { and } \beta_{2}=\frac{1}{3 k-1} .
$$

If $E_{i}=24 D_{i}$, then $\left(\frac{l_{i}}{D_{i}}\right)^{\alpha_{2}} n^{-\beta_{2}} \leq 24 D_{i}$. In this case, the first term in the righthand side of (4.15) controls the second one. Otherwise, both terms are equal up to a constant factor. We deduce from (4.15) that

$$
I\left(\mathcal{Q}_{i} \backslash \mathcal{Q}_{i, 0}, \mathcal{H}\right)= \begin{cases}O\left(n D_{i}^{3}+l_{i}\right) & \text { if } E_{i}=24 D_{i}, \\ O\left(n^{1-2 \beta_{2}} l_{i}^{2 \alpha_{2}} D_{i}^{1-2 \alpha_{2}}+l_{i}\right) & \text { otherwise }\end{cases}
$$

By (4.9),

$$
\sum_{i} n D_{i}^{3} \leq n D^{3}=O\left(m^{1-\frac{k-1}{4 k-1}} n^{1-\frac{3}{4 k-1}}+n\right),
$$

as the term $n D^{3}$ appears in (4.6) and is accounted for in (4.8). Using the Hölder inequality as well as (4.9) and (4.10), we get

$$
\begin{aligned}
\sum_{i} n^{1-2 \beta_{2}} l_{i}^{2 \alpha_{2}} D_{i}^{1-2 \alpha_{2}} \leq n^{1-2 \beta_{2}}\left(\sum_{i} l_{i}\right)^{2 \alpha_{2}}\left(\sum_{i} D_{i}\right)^{1-2 \alpha_{2}} & \\
& \leq n^{1-2 \beta_{2}} m_{0}^{2 \alpha_{2}} D^{1-2 \alpha_{2}} .
\end{aligned}
$$

We now substitute the value of $D$ from (4.7) and those of $\alpha_{1}, \alpha_{2}, \beta_{1}$ and $\beta_{2}$ in the above expression. If $D=24$, then $m^{k}=O(n)$ and so $n^{1-2 \beta_{2}} m_{0}^{2 \alpha_{2}} D^{1-2 \alpha_{2}}=$ $n^{1-2 \beta_{2}} m_{0}^{2 \alpha_{2}}=O(n)$. Otherwise,

$$
n^{1-2 \beta_{2}} m_{0}^{2 \alpha_{2}} D^{1-2 \alpha_{2}} \leq n^{1-2 \beta_{2}} m^{2 \alpha_{2}}\left(m^{\alpha_{1}} n^{-\beta_{1}}\right)^{1-2 \alpha_{2}}=m^{1-\frac{k-1}{4 k-1}} n^{1-\frac{3}{4 k-1}} .
$$


It follows from (4.17), (4.18), (4.19), (4.20) and (4.10) that

$$
\begin{aligned}
I\left(\mathcal{P}_{0} \backslash \bigcup_{i} \mathcal{Q}_{i, 0}, \mathcal{H}\right) & =\sum_{i} I\left(\mathcal{Q}_{i} \backslash \mathcal{Q}_{i, 0}, \mathcal{H}\right) \\
& =O\left(\sum_{i} n D_{i}^{3}+\sum_{i} n^{1-2 \beta_{2}} l_{i}^{2 \alpha_{2}} D_{i}^{1-2 \alpha_{2}}+\sum_{i} l_{i}\right) \\
& =O\left(m^{1-\frac{k-1}{4 k-1}} n^{1-\frac{3}{4 k-1}}+n+m_{0}\right) .
\end{aligned}
$$

Third level partitioning. For each $i \in I$, let $W_{i}=\bigcup_{j \in J_{i}} W_{i, j}$ be the decomposition of the surface $W_{i}=V\left(f_{i}, g_{i}\right)$ into irreducible components. Set $\Delta_{i, j}=\operatorname{deg}\left(W_{i, j}\right)$ for each $j$. By Bézout's inequality,

$$
\sum_{j \in J_{i}} \Delta_{i, j}=\operatorname{deg}\left(W_{i}\right) \leq D_{i} E_{i}
$$

We denote by $W_{i}(\mathbb{R})_{0}$ and $W_{i, j}(\mathbb{R})_{0}$ the set of isolated points of the semi-algebraic sets $W_{i}(\mathbb{R})$ and $W_{i, j}(\mathbb{R})$, respectively. We then choose an arbitrary partition of the set $\mathcal{Q}_{i, 0}=\mathcal{Q}_{i} \cap W_{i}$ into disjoints subsets $\mathcal{R}_{i, j}, j \in J_{i}$, such that

$$
\mathcal{R}_{i, j} \subset W_{i, j}(\mathbb{R}) \quad \text { and } \quad \mathcal{R}_{i, j} \cap W_{i, j}(\mathbb{R})_{0} \subset W_{i}(\mathbb{R})_{0} .
$$

Set $e_{i, j}=\operatorname{card}\left(\mathcal{R}_{i, j}\right)$ for each $j$. Then

$$
\sum_{j} e_{i, j}=l_{i, 0}
$$

Let $j \in J_{i}$. Being an irreducible component of $W_{i}=V\left(f_{i}, g_{i}\right)$, the variety $W_{i, j}$ is partially defined at degree $E_{i}$. Let $F_{i, j} \geq 24 E_{i}$, to be fixed later on. By Theorem 3.1, there exists $h_{i, j} \in \mathbb{R}\left[x_{1}, x_{2}, x_{3}, x_{4}\right]_{\leq F_{i, j}}$ such that $\operatorname{dim}\left(W_{i, j} \cap V\left(h_{i, j}\right)\right)=1$ and, for each $\eta \in\{ \pm 1\}^{\operatorname{irr}\left(h_{i, j}\right)}$,

$$
\operatorname{card}\left(\mathcal{R}_{i, j}(\eta)\right)=O\left(\frac{e_{i, j}}{\Delta_{i, j} F_{i, j}^{2}}\right) .
$$

Similarly as before, choose a minimal subset $\Sigma_{3, i, j} \subset\{ \pm 1\}^{\operatorname{irr}\left(h_{i, j}\right)}$ realizing all nonempty subsets of the form $\mathcal{R}_{i, j}(\eta)$.

Consider the curve $Y_{i, j}=W_{i, j} \cap V\left(h_{i, j}\right)$ and partition $\mathcal{R}_{i, j}$ into the disjoint subsets $\mathcal{R}_{i, j, 0}=\mathcal{R}_{i, j} \cap Y_{i, j}$ and $\mathcal{R}_{i, j}(\eta), \eta \in \Sigma_{3, i, j}$. Set also $e_{i, j, 0}=\operatorname{card}\left(\mathcal{R}_{i, j, 0}\right)$ and $e_{i, j, \eta}=\operatorname{card}\left(\mathcal{R}_{i, j}(\eta)\right)$ for each $\eta$. Hence,

$$
e_{i, j, 0}+\sum_{\eta \in \Sigma_{3, i, j}} e_{i, j, \eta}=e_{i, j} .
$$

We first bound the number of incidences of $\mathcal{R}_{i, j} \backslash \mathcal{R}_{i, j, 0}$ with hypersurfaces that contain at least $k$ points in some $\mathcal{R}_{i, j}(\eta)$. Similarly as for (4.3) and (4.12), the hypothesis (c) implies that, for each $\eta$,

$$
I_{\geq k}\left(\mathcal{R}_{i, j}(\eta), \mathcal{H}\right) \leq c k\left(\begin{array}{c}
e_{i, j, \eta} \\
k
\end{array}\right)=O\left(e_{i, j, \eta}^{k}\right) .
$$

By Proposition 2.6, after dehomogenizing, there are coprime polynomials $\widetilde{f}_{i, j}, \widetilde{g}_{i, j} \in$ $\mathbb{R}\left[x_{1}, x_{2}, x_{3}, x_{4}\right]$ such that $W_{i, j}$ is an irreducible component of the variety $\widetilde{W}_{i, j}=$ $V\left(\widetilde{f}_{i, j}, \widetilde{g}_{i, j}\right)$ and

$$
\operatorname{deg}\left(\widetilde{f}_{i, j}\right) \operatorname{deg}\left(\widetilde{g}_{i, j}\right)=O\left(\Delta_{i, j}\right)
$$


Since $W_{i, j}$ is partially defined at degree $E_{i}$, we can furthermore deduce that $\operatorname{deg}\left(\widetilde{f}_{i, j}\right), \operatorname{deg}\left(\widetilde{g}_{i, j}\right) \leq$ $E_{i}$.

The number of nonempty subsets of the form $\mathcal{R}_{i, j}(\eta)$ is bounded by the number of connected components of $\widetilde{W}_{i, j}(\mathbb{R}) \backslash V\left(h_{i, j}\right)$, as explained in Remark 3.2. By Theorem 2.8 and (4.26), this number of connected components is bounded by

$$
\mathrm{b}_{0}\left(\widetilde{W}_{i, j}(\mathbb{R}) \backslash V\left(h_{i, j}\right)\right)=O\left(\operatorname{deg}\left(\widetilde{f}_{i, j}\right) \operatorname{deg}\left(\widetilde{g}_{i, j}\right) F_{i, j}^{2}\right)=O\left(\Delta_{i, j} F_{i, j}^{2}\right) .
$$

By (4.24), (4.25) and (4.27),

$$
\sum_{\eta} I_{\geq k}\left(\mathcal{R}_{i, j}(\eta), \mathcal{H}\right)=O\left(\sum_{\eta}\left(\frac{e_{i, j}}{\Delta_{i, j} F_{i, j}^{2}}\right)^{k}\right)=O\left(e_{i, j}^{k} \Delta_{i, j}^{1-k} F_{i, j}^{2-2 k}\right) .
$$

We now bound the number of incidences of $\mathcal{R}_{i, j} \backslash \mathcal{R}_{i, j, 0}$ with hypersurfaces that contain at most $k-1$ points in every $\mathcal{R}_{i, j}(\eta)$. We would like to use an argument similar to those used above in the case of first and second level partitioning, and bound, for each $H \in \mathcal{H}$, the number of these incidences on $H$, by $k$ times the number of connected components of $\mathbb{R}^{4} \backslash V\left(h_{i, j}\right)$ having nonempty intersection with $H \cap W_{i, j}(\mathbb{R})$ using Theorem 2.8. However, there are two difficulties in this approach. First, unlike the prior cases, we do not have the equations defining $W_{i, j}$, but rather those of a possibly larger variety $\widetilde{W}_{i, j}$. This is not a serious problem, since clearly the number of connected components of $\mathbb{R}^{4} \backslash V\left(h_{i, j}\right)$, met by the possibly larger set $H \cap \widetilde{W}_{i, j}(\mathbb{R})$ is an upper bound on the number of connected components of $\mathbb{R}^{4} \backslash V\left(h_{i, j}\right)$ having nonempty intersection with $H \cap W_{i, j}(\mathbb{R})$. The second difficulty is more serious. To apply Theorem 2.8 to obtain a sufficiently good upper bound (see (4.30) below) we require that the dimension of the intersection $H \cap \widetilde{W}_{i, j}$ is one (if $H \cap \widetilde{W}_{i, j}$ is non-empty), and this requirement might not be satisfied. To circumvent this difficulty, for each $H \in \mathcal{H}$, we partition $W_{i, j}(\mathbb{R})$ into two semialgebraic subsets, namely $G_{i, j}(H)$ and $B_{i, j}(H)$. The non-isolated points of $W_{i, j}(\mathbb{R})$ which belong $B_{i, j}(H)$ are points having an open neighborhood in $W_{i, j}(\mathbb{R})$ (with respect to its Euclidean topology) which is contained also in $H$. Such points have the bad property that the intersection of $W_{i, j}(\mathbb{R})$ with a small perturbation of $H$ could be empty in a neighborhood of such a point. We bound incidences created by points in the various $B_{i, j}(H)$ using a separate argument (see inequality (4.40) below).

On the other hand, to bound the incidences in $H \cap G_{i, j}(H)$, we show (using Proposition 2.9) that it is possible to replace $H$ by a slightly perturbed hypersurface $\widetilde{H} \subset \mathbb{R}^{4}$ of degree twice the degree of $H$, satisfying:

(a) every connected component of $\mathbb{R}^{4} \backslash V\left(h_{i, j}\right)$ having nonempty intersection with $H \cap G_{i, j}(H)$ also has a non-empty intersection with $\widetilde{H} \cap \widetilde{W}_{i, j}(\mathbb{R})$;

(b) the dimension of $\widetilde{H} \cap \widetilde{W}_{i, j}$ is equal to 1 if $\widetilde{H} \cap \widetilde{W}_{i, j} \neq \emptyset$.

This, allows us to obtain the necessary estimate (see (4.30) below) on the number of connected components of $\mathbb{R}^{4} \backslash V\left(h_{i, j}\right)$ having nonempty intersection with $H \cap$ $G_{i, j}(H)$ using Theorem 2.8. We now make the above arguments precise as follows.

Given $H \in \mathcal{H}$, we denote by $B_{i, j}(H) \subset W_{i, j}(\mathbb{R})$ the semi-algebraic subset of points $p \in W_{i, j}(\mathbb{R})$ having an open neighborhood, in the Euclidean topology of $W_{i, j}(\mathbb{R})$, contained in $H$. We also set $G_{i, j}(H)=W_{i, j}(\mathbb{R}) \backslash B_{i, j}(H)$. Notice that unlike $B_{i, j}(H)$, the semi-algebraic set $G_{i, j}(H)$ is not necessarily contained in $H$, and that $W_{i, j}(\mathbb{R})_{0} \cap H \subset B_{i, j}(H)$. 
For any finite subset $\mathcal{R} \subset W_{i, j}(\mathbb{R})$ we set

$$
\mathcal{I}^{\mathrm{B}}(\mathcal{R}, \mathcal{H})=\bigcup_{H \in \mathcal{H}} \mathcal{I}\left(\mathcal{R} \cap B_{i, j}(H), \mathcal{H}\right) \quad \text { and } \quad \mathcal{I}^{\mathrm{G}}(\mathcal{R}, \mathcal{H})=\bigcup_{H \in \mathcal{H}} \mathcal{I}\left(\mathcal{R} \cap G_{i, j}(H), \mathcal{H}\right) .
$$

We also set $I^{\mathrm{B}}(\mathcal{R}, \mathcal{H})=\operatorname{card}\left(\mathcal{I}^{\mathrm{B}}(\mathcal{R}, \mathcal{H})\right)$ and $I^{\mathrm{G}}(\mathcal{R}, \mathcal{H})=\operatorname{card}\left(\mathcal{I}^{\mathrm{G}}(\mathcal{R}, \mathcal{H})\right)$. Clearly,

$$
I(\mathcal{R}, \mathcal{H})=I^{\mathrm{B}}(\mathcal{R}, \mathcal{H})+I^{\mathrm{G}}(\mathcal{R}, \mathcal{H})
$$

We first treat the incidences in $G_{i, j}(H)$. Write $H=V(b)$ with $b \in \mathbb{R}\left[x_{1}, x_{2}, x_{3}, x_{4}\right]$. The number of nonempty subsets of the form $\mathcal{R}_{i, j}(\eta) \cap G_{i, j}(H)$ is bounded by the number of connected components of $\mathbb{R}^{4} \backslash V\left(h_{i, j}\right)$ having nonempty intersection with $H \cap G_{i, j}(H)$. By Proposition 2.9, this number of connected components is bounded by the number of connected components of the semi-algebraic set

$$
\left(\widetilde{W}_{i, j} \cap V\left(b^{2}-\varepsilon\right)\right)(\mathbb{R}) \backslash V\left(h_{i, j}\right)=V\left(b^{2}-\varepsilon, \widetilde{f}_{i, j}, \widetilde{g}_{i, j}\right)(\mathbb{R}) \backslash V\left(h_{i, j}\right)
$$

for any $\varepsilon>0$ sufficiently small. Choosing a possibly smaller $\varepsilon>0$, we also have that $\operatorname{dim}\left(\widetilde{W}_{i, j} \cap V\left(b^{2}-\varepsilon\right)\right)=1$ if $\widetilde{W}_{i, j} \cap V\left(b^{2}-\varepsilon\right) \neq \emptyset$. To see this, observe that the set of critical values of $b^{2}$ restricted to $\operatorname{reg}\left(\widetilde{W}_{i, j}\right)$ is finite using Sard's theorem [Łoj91, page 255], and hence, for all $\varepsilon>0$ small enough, $\varepsilon$ is a regular value of $b^{2}$ restricted to $\operatorname{reg}\left(\widetilde{W}_{i, j}\right)$. It follows that either $\widetilde{W}_{i, j} \cap V\left(b^{2}-\varepsilon\right)$ is empty, or $\operatorname{dim}\left(\widetilde{W}_{i, j} \cap V\left(b^{2}-\varepsilon\right)\right)=1$, using the implicit function theorem (see for example [GH78, page 19]).

For any such choice of $\varepsilon$, by Theorem 2.8 and (4.26), the number of connected components of the semi-algebraic set in (4.29) is bounded by

$$
O\left(\operatorname{deg}\left(b^{2}-\varepsilon\right) \operatorname{deg}\left(\widetilde{f}_{i, j}\right) \operatorname{deg}\left(\widetilde{g}_{i, j}\right) \operatorname{deg}\left(h_{i, j}\right)\right)=O\left(\Delta_{i, j} F_{i, j}\right) .
$$

Thus

$$
\sum_{\eta} I_{<k}^{\mathrm{G}}\left(\mathcal{R}_{i, j}(\eta), \mathcal{H}\right)=O\left(n \Delta_{i, j} F_{i, j}\right)
$$

where

$$
I_{<k}^{\mathrm{G}}\left(\mathcal{R}_{i, j}(\eta), \mathcal{H}\right)=\operatorname{card}\left(\mathcal{I}^{\mathrm{G}}\left(\mathcal{R}_{i, j}(\eta), \mathcal{H}\right) \cap \mathcal{I}_{<k}\left(\mathcal{R}_{i, j}(\eta), \mathcal{H}\right)\right) .
$$

Gathering together (4.28) and (4.31), we obtain that

$$
\begin{aligned}
I^{\mathrm{G}}\left(\mathcal{R}_{i, j} \backslash \mathcal{R}_{i, j, 0}, \mathcal{H}\right)=\sum_{\eta} I^{\mathrm{G}}\left(\mathcal{R}_{i, j}(\eta), \mathcal{H}\right) & \\
& =O\left(n \Delta_{i, j} F_{i, j}+e_{i, j}^{k} \Delta_{i, j}^{1-k} F_{i, j}^{2-2 k}\right) .
\end{aligned}
$$

We set

$$
F_{i, j}=\max \left(24 E_{i},\left(\frac{e_{i, j}}{\Delta_{i, j}}\right)^{\alpha_{3}} \frac{1}{n^{\beta_{3}}}\right) \quad \text { with } \alpha_{3}=\frac{k}{2 k-1} \text { and } \beta_{3}=\frac{1}{2 k-1} .
$$

If $F_{i, j}=24 E_{i}$, then $\left(\frac{e_{i, j}}{\Delta_{i, j}}\right)^{\alpha_{3}} n^{-\beta_{3}}=O\left(E_{i}\right)$. In this case, the first term in the right-hand side of (4.32) controls the second one and, otherwise, both terms are equal up to a constant factor. Hence,

$$
I^{\mathrm{G}}\left(\mathcal{R}_{i, j} \backslash \mathcal{R}_{i, j, 0}, \mathcal{H}\right)= \begin{cases}O\left(n \Delta_{i, j} E_{i}\right) & \text { if } F_{i, j}=24 E_{i}, \\ O\left(n^{1-\beta_{3}} e_{i, j}^{\alpha_{3}} \Delta_{i, j}^{1-\alpha_{3}}\right) & \text { otherwise. }\end{cases}
$$


By (4.22) and Bézout's inequality,

$$
\sum_{i, j} n \Delta_{i, j} E_{i} \leq \sum_{i} n D_{i} E_{i}^{2}=O\left(m^{1-\frac{k-1}{4 k-1}} n^{1-\frac{3}{4 k-1}}+n\right),
$$

as shown when passing from (4.15) to (4.21). Else, applying the Hölder inequality together with (4.23) and (4.9),

$$
\begin{aligned}
\sum_{i, j} n^{1-\beta_{3}} e_{i, j}^{\alpha_{3}} \Delta_{i, j}^{1-\alpha_{3}} \leq n^{1-\beta_{3}}\left(\sum_{i, j} e_{i, j}\right)^{\alpha_{3}} & \left(\sum_{i, j} \Delta_{i, j}\right)^{1-\alpha_{3}} \\
& \leq n^{1-\beta_{3}} m^{\alpha_{3}}\left(\sum_{i} D_{i} E_{i}\right)^{1-\alpha_{3}} .
\end{aligned}
$$

Recall that $E_{i}=\max \left(24 D_{i},\left(\frac{l_{i}}{D_{i}}\right)^{\alpha_{2}} n^{-\beta_{2}}\right)$ as in (4.16). Hence

$$
\begin{aligned}
\sum_{i} D_{i} E_{i} & =O\left(\sum_{i} D_{i}^{2}+n^{-\beta_{2}} \sum_{i} l_{i}{ }^{\alpha_{2}} D_{i}{ }^{1-\alpha_{2}}\right) \\
& =O\left(\sum_{i} D_{i}^{2}+n^{-\beta_{2}}\left(\sum_{i} l_{i}\right)^{\alpha_{2}}\left(\sum_{i} D_{i}\right)^{1-\alpha_{2}}\right) \\
& =O\left(D^{2}+n^{-\beta_{2}} m^{\alpha_{2}} D^{1-\alpha_{2}}\right) .
\end{aligned}
$$

Recall also that $D=\max \left(24, m^{\alpha_{1}} n^{-\beta_{1}}\right)$ as in (4.7). If $D=24$, then $m^{k}=O(n)$. In this case, $\sum_{i} D_{i} E_{i}=O(1)$. Otherwise, substituting $D=m^{\alpha_{1}} n^{-\beta_{1}}$ in (4.36) and the sum $\sum_{i} D_{i} E_{i}$ into (4.35),

$$
\begin{aligned}
n^{1-\beta_{3}} m^{\alpha_{3}}\left(\sum_{i} D_{i} E_{i}\right)^{1-\alpha_{3}} & =O\left(n^{1-\beta_{3}} m^{\alpha_{3}}\left(n^{-\beta_{2}} m^{\alpha_{2}}\left(m^{\alpha_{1}} n^{-\beta_{1}}\right)^{1-\alpha_{2}}\right)^{1-\alpha_{3}}\right) \\
& =O\left(m^{1-\frac{k-1}{4 k-1}} n^{1-\frac{3}{4 k-1}}\right) .
\end{aligned}
$$

It follows from (4.33), (4.34), (4.35), (4.36) and(4.37), that

$$
\begin{aligned}
I^{\mathrm{G}}\left(\bigcup_{i} \mathcal{Q}_{i, 0} \backslash \bigcup_{i, j} \mathcal{R}_{i, j, 0}, \mathcal{H}\right) & =\sum_{i, j} I^{\mathrm{G}}\left(\mathcal{R}_{i, j} \backslash \mathcal{R}_{i, j, 0}, \mathcal{H}\right) \\
& =O\left(\sum_{i, j}\left(n \Delta_{i, j} F_{i, j}+e_{i, j}^{k} \Delta_{i, j}^{1-k} F_{i, j}^{2-2 k}\right)\right) \\
& =O\left(m^{1-\frac{k-1}{4 k-1}} n^{1-\frac{3}{4 k-1}}+n\right) .
\end{aligned}
$$

Finally, we treat the incidences in $B_{i, j}(H)$. We claim that for each $p \in \mathcal{R}_{i, j} \backslash$ $W_{i}(\mathbb{R})_{0}$ there are at most 3 hypersurfaces in $\mathcal{H}$ such that $p \in B_{i, j}(H)$. To see this, observe that $p \in B_{i, j}(H)$ implies that $H$ contains an open neighborhood $U \subset W_{i, j}(\mathbb{R})$ of $p$. Since $p$ is not an isolated point of $W_{i, j}(\mathbb{R})$, if follows that $U$ is of real dimension at least 1 . The claim then follows from the hypothesis (b).

Hence,

$$
I^{\mathrm{B}}\left(\mathcal{R}_{i, j} \backslash W_{i}(\mathbb{R})_{0}, \mathcal{H}\right) \leq 3 e_{i, j} .
$$

The incidences of $\mathcal{Q}_{i}$ with hypersurfaces $H \in \mathcal{H}$ containing $V_{i}$ are already accounted for in (4.14). Hence, we can suppose that $V_{i}$ is not contained in $H$. In this case, by Theorem $2.8, \operatorname{card}\left(H \cap W_{i}(\mathbb{R})_{0}\right) \leq \mathrm{b}_{0}\left(V\left(b, f_{i}, g_{i}\right)\right)=O\left(D_{i} E_{i}^{2}\right)$, where $b$ is the polynomial defining $H$. Together with (4.14), this implies that

$$
\sum_{j} I^{\mathrm{B}}\left(\mathcal{R}_{i, j} \cap W_{i}(\mathbb{R})_{0}, \mathcal{H}\right)=O\left(n D_{i} E_{i}^{2}+l_{i}\right)
$$


It follows from (4.40), (4.23) and (4.41) that

$$
\sum_{j} I^{\mathrm{B}}\left(\mathcal{R}_{i, j}, \mathcal{H}\right)=O\left(n D_{i} E_{i}^{2}+l_{i}\right)+O\left(l_{i, 0}\right)=O\left(n D_{i} E_{i}^{2}+l_{i}\right) .
$$

This bound already appears in (4.15). The contribution of the sum of these terms over $i \in I$ is accounted for in (4.21) and can be absorbed into the bound (4.39), after adding the term $m$. We conclude that

$$
I\left(\bigcup_{i} \mathcal{Q}_{i, 0} \backslash \bigcup_{i, j} \mathcal{R}_{i, j, 0}, \mathcal{H}\right)=O\left(m^{1-\frac{k-1}{4 k-1}} n^{1-\frac{3}{4 k-1}}+m+n\right) .
$$

The case of curves and conclusion of the proof. Finally, we bound the number of incidences that occur on the curves $Y_{i, j}=W_{i, j} \cap V\left(h_{i, j}\right)$.

For each $i, j$, set $\mathcal{R}_{i, j, 0}=\mathcal{R}_{i, j} \cap Y_{i, j}$. Let $Y_{i, j}=\bigcup_{l \in L_{i, j}} Y_{i, j, l}$ be the decomposition of $Y_{i, j}$ into irreducible components and consider an arbitrary partition of $\mathcal{R}_{i, j, 0}$ into disjoint subsets $\mathcal{S}_{i, j, l}, l \in L_{i, j}$, with $\mathcal{S}_{i, j, l} \subset Y_{i, j, l}$ for all $l$.

Let $l \in L_{i, j}$ and $H \in \mathcal{H}$. If $Y_{i, j, l}$ is not contained in $H$, then the number of incidences between $\mathcal{S}_{i, j, l}$ and this hypersurface is bounded by $\operatorname{card}\left(Y_{i, j, l} \cap H\right)$. From Bézout's inequality, we deduce that

$$
I\left(\mathcal{S}_{i, j, l},\{H\}\right) \leq \begin{cases}\operatorname{deg}(H) \operatorname{deg}\left(Y_{i, j, l}\right) & \text { if } Y_{i, j, l} \not \subset H, \\ \operatorname{card}\left(\mathcal{S}_{i, j, l}\right) & \text { if } Y_{i, j, l} \subset H .\end{cases}
$$

The hypothesis (b) implies that, for each $l$, there are at most 3 hypersurfaces in $\mathcal{H}$ containing $Y_{i, j, l}$. It follows from (4.43) that

$$
\begin{aligned}
I\left(\mathcal{R}_{i, j, 0}, \mathcal{H}\right) & =\sum_{l \in L_{i, j}} \sum_{H \in \mathcal{H}} I\left(\mathcal{S}_{i, j, l},\{H\}\right) \\
& =O\left(\sum_{l, H} \operatorname{deg}\left(Y_{i, j, l}\right)\right)+3 \sum_{l} \operatorname{card}\left(\mathcal{S}_{i, j, l}\right) \\
& =O\left(n \operatorname{deg}\left(Y_{i, j}\right)+\operatorname{card}\left(\mathcal{R}_{i, j, 0}\right)\right) .
\end{aligned}
$$

By Bézout's inequality, $\operatorname{deg}\left(Y_{i, j}\right) \leq \Delta_{i, j} F_{i, j}$. Using (4.44),

$$
I\left(\mathcal{P} \cap \bigcup_{i, j} Y_{i, j}, \mathcal{H}\right)=\sum_{i, j} I\left(\mathcal{R}_{i, j, 0}, \mathcal{H}\right)=O\left(\sum_{i, j} n \Delta_{i, j} F_{i, j}+\sum_{i, j} \operatorname{card}\left(\mathcal{R}_{i, j, 0}\right)\right) .
$$

The first sum in the right-hand side of (4.45) appears in (4.38) and is already accounted for in (4.39). By construction, the family of sets $\left\{\mathcal{R}_{i, j, 0}\right\}_{i, j}$ is a partition of $\mathcal{P} \cap \bigcup_{i, j} Y_{i, j}$. Therefore, the sum of their cardinalities is bounded by $m$. Hence,

$$
I\left(\mathcal{P} \cap \bigcup_{i, j} Y_{i, j}, \mathcal{H}\right)=O\left(m^{1-\frac{k-1}{4 k-1}} n^{1-\frac{3}{4 k-1}}+m+n\right) .
$$

The statement now follows by summing up the contributions from (4.8), (4.21), (4.42) and (4.46).

We close this paper by proposing the next conjecture on the number of pointhypersurfaces incidences in higher dimension.

Conjecture 4.1. Let $d, k, c \geq 1$, and let $\mathcal{P}$ be a finite set of points of $\mathbb{R}^{d}$ and $\mathcal{H} a$ finite set of hypersurfaces of $\mathbb{C}^{d}$ satisfying the following conditions:

(a) the degrees of the hypersurfaces in $\mathcal{H}$ are bounded by $c$;

(b) the intersection of any family of d distinct hypersurfaces in $\mathcal{H}$ is finite; 
(c) for any subset of $k$ distinct points in $\mathcal{P}$, the number of hypersurfaces in $\mathcal{H}$ containing them is bounded by $c$.

Set $m=\operatorname{card}(\mathcal{P})$ and $n=\operatorname{card}(\mathcal{H})$. Then

$$
I(\mathcal{P}, \mathcal{H})=O_{d, k, c}\left(m^{1-\frac{k-1}{d k-1}} n^{1-\frac{d-1}{d k-1}}+m+n\right) .
$$

This conjecture is suggested by the bound that follows from the first level of the polynomial partitioning method applied to this problem. It contains the statements of the Szemerédi-Trotter theorem 1.4, the results of Zahl and Kaplan, Matoušek, Sharir and Safernová in three dimensions [Zah13, KMSS12], and Theorem 1.5.

Concurrently with this paper, a proof of a weaker version of this conjecture, with an extra factor of $m^{\varepsilon}$ in the bound, has appeared in [FPS $\left.{ }^{+} 14\right]$.

\section{REFERENCES}

[BB12] S. Barone and S. Basu, Refined bounds on the number of connected components of sign conditions on a variety, Discrete Comput. Geom. 47 (2012), 577-597. 4, 9

[BB13] S. Barone and S. Basu, On a real analogue of Bezout inequality and the number of connected components of sign conditions, ArXiv e-prints (2013). 4, 9

[BPR06] S. Basu, R. Pollack, and M.-F. Roy, Algorithms in real algebraic geometry, second ed., Algorithms Comput. Math., vol. 10, Springer-Verlag, 2006. 10

$\left[\mathrm{CEG}^{+} 90\right]$ K. Clarkson, H. Edelsbrunner, L.J. Guibas, M. Sharir, and E. Welzl, Combinatorial complexity bounds for arrangements of curves and spheres, Discrete Comput. Geom. 5 (1990), 99-160. 1

[Cha89] M. Chardin, Une majoration de la fonction de Hilbert et ses conséquences pour l'interpolation algébrique, Bull. Soc. Math. France 117 (1989), 305-318. 3, 7

[CP99] M. Chardin and P. Philippon, Régularité et interpolation, J. Algebraic Geom. 8 (1999), 471-481. 3, 7

[ES11] G. Elekes and M. Sharir, Incidences in three dimensions and distinct distances in the plane, Combin. Probab. Comput. 20 (2011), 571-608. 1

$\left[\right.$ FPS $\left.^{+} 14\right]$ J. Fox, J. Pach, A. Sheffer, A. Suk, and J. Zahl, A semi-algebraic version of Zarankiewicz's problem, e-print arXiv:1407.5705v1, 2014. 4, 22

[Ful84] W. Fulton, Intersection theory, Ergeb. der Math. und ihrer Grenzgebiete, vol. 3, Springer-Verlag, 1984. 4

[GH78] Phillip Griffiths and Joseph Harris, Principles of algebraic geometry, WileyInterscience [John Wiley \& Sons], New York, 1978, Pure and Applied Mathematics. MR 507725 (80b:14001) 19

[GK10] L. Guth and N. Katz, On the Erdös distinct distance problem in the plane, e-print arXiv:1011.4105v3, 2010. 1, 2, 11, 13

[Har77] R. Hartshorne, Algebraic geometry, Graduate Texts in Math., vol. 52, Springer-Verlag, 1977. 16

[KMS12] H. Kaplan, J. Matoušek, and M. Sharir, Simple proofs of classical theorems in discrete geometry via the Guth-Katz polynomial partitioning technique, Discrete Comput. Geom. 48 (2012), 499-517. 1

[KMSS12] H. Kaplan, J. Matoušek, M. Sharir, and S. Safernová, Unit distances in three dimensions, Combinat. Probab. Comput. 21 (2012), 597-610. 2, 3, 4, 11, 13, 22

[Łoj91] Stanisław Łojasiewicz, Introduction to complex analytic geometry, Birkhäuser Verlag, Basel, 1991, Translated from the Polish by Maciej Klimek. MR 1131081 (92g:32002) 19

[Mil64] J. Milnor, On the Betti numbers of real varieties, Proc. Amer. Math. Soc. 15 (1964), 275-280. 2, 9

[MS14] J. Matousek and Z. Safernova, Multilevel polynomial partitions and simplified range searching, ArXiv e-prints (2014). 3

[Nes84] Yu. V. Nesterenko, Estimates for the characteristic function of a prime ideal, Mat. Sb. (N.S.) 123(165) (1984), 11-34. 7

[PO49] I. G. Petrovskiǔ and O. A. Oleŭnik, On the topology of real algebraic surfaces, Izvestiya Akad. Nauk SSSR. Ser. Mat. 13 (1949), 389-402. 2, 9 
[PS98] J. Pach and M. Sharir, On the number of incidences between points and curves, Combin. Probab. Comput. 7 (1998), 121-127. 3

[She14] A. Sheffer, Incidences in d-dimensional spaces, two blogs posted at http:// adamsheffer.wordpress.com, 2014. 4

[Som14] M. Sombra, Bounds for the Hilbert function of polynomial ideals, talk at the IPAM workshop "Tools from Algebraic Geometry", https://www.ipam.ucla.edu/schedule. aspx?pc=ccgws $2,2014.4,7$

[ST83] E. Szemerédi and W. T. Trotter, Jr., Extremal problems in discrete geometry, Combinatorica 3 (1983), 381-392. 3

[ST12] J. Solymosi and T. Tao, An incidence theorem in higher dimensions, Discrete Comput. Geom. 48 (2012), 255-280. 1

[Tho65] R. Thom, Sur l'homologie des variétés algébriques réelles, Differential and Combinatorial Topology (A Symposium in Honor of Marston Morse), Princeton Univ. Press, 1965, pp. 255-265. 2, 9

[Zah13] J. Zahl, An improved bound on the number of point-surface incidences in three dimensions, Contrib. Discrete Math. 8 (2013), 100-121. 2, 3, 4, 11, 13, 22

Department of Mathematics, Purdue University. West Lafayette, IN 47906, U.S.A.

E-mail address: sbasu@math.purdue.edu

URL: http://www . math. purdue. edu/ sbasu

icRea \& Departament d’Àlgebra i Geometria, Universitat de Barcelona. Gran Via 585, 08007 Barcelona, Spain

E-mail address: sombra@ub.edu

$U R L$ : http://atlas.mat.ub.es/personals/sombra 